\title{
Anatomically distinct OFC-PCC circuits relay choice from value space to action space
}

Department of Neuroscience,

Center for Magnetic Resonance Research, and

Department of Biomedical Engineering

University of Minnesota, Minneapolis MN 55455

\section{Corresponding author:}

Maya Zhe Wang

Department of Neuroscience

University of Minnesota, Minneapolis MN 55455

Email: mayawangz@gmail.com

\section{Funding statement}

This research was supported by NIH grants R01 DA038106 (to BYH), R01 MH 118257 (to SRH), and a MNDrive fellowship (to MZW)

\section{Competing interests}

The authors have no competing interests to declare.

\section{Acknowledgements}

We thank Giuliana Loconte, Hannah Lee, Tanya Casta, Mark Grier, Megan Monko, and Adriana Cushnie for experimental help. 


\section{ABSTRACT}

39 Economic choice necessarily involves the transformation of abstract, object-based

40 representations to concrete, action-based ones. This transformation is both determined and

41 delimited by the neuroanatomical organization of the regions that implement it. In choice, the

42 orbitofrontal cortex (OFC) plays a key role in both abstract valuation and cognitive mapping.

43 However, determining the neural processes underlying this transformation has proven difficult.

44 We hypothesized that difficulty stems from in part from the fact that the OFC consists of multiple

45 functionally distinct zones that are distinguished by their differing contributions to the abstract-

46 concrete transformation, and that these functions reflect their differing long-range projections.

47 Here we identify two such subregions, defined by stronger or weaker bidirectional anatomical

48 connectivity with the posterior cingulate cortex (PCC). We call these regions OFCin and OFCout,

49 respectively. We find that OFCin, relative to OFCout, shows enhanced functional connectivity

50 with PCC, as indicated by both spike-field coherence and mutual information. We find

51 converging evidence that the OFCin-PCC circuit, but not the OFCout-PCC circuit, relays choice

52 signals from an abstract value space to a concrete action space. Moreover, the OFCin-PCC circuit

53 shows a putative bidirectional mutually excitatory pattern. Together, these results support the

54 hypothesis that OFC-PCC subareal organization is critical for understanding the implementation

55 of offer-action transformation in economic choice. 
Among brain regions associated with economic choice, the orbitofrontal cortex (OFC)

59 has attracted the lion's share of attention (Bradfield \& Hart, 2020; Kaplan et al., 2017; Padoa-

60 Schioppa \& Conen, 2017; Schoenbaum et al., 2009; Stalnaker et al., 2015; Wallis, 2007;

61 Wikenheiser \& Schoenbaum, 2016; Wilson et al., 2014; Rudebeck and Murray, 2014 and 2018).

62 This region is associated with evaluation, value comparison, cognitive mapping, and prospection

63 (Padoa-Schioppa, 2011; Rushworth et al., 2011; Schuck et al., 2016; Wallis, 2007; Wang et al.,

64 2020; Wang \& Hayden, 2017). Consequently, OFC is seen as playing a central role in

65 choice. Furthermore, there is increasing attention being paid to functionally unique subdivisions

66 of the OFC (Rudebeck \& Murray, 2011 and 2018). For example, the medial OFC may be more

67 associated with abstract valuation and learning processes (Noonan et al., 2010; Rushworth et al.,

68 2011; Levy \& Glimcher, 2014), whereas central OFC may help to associate stimuli with

69 outcomes or signal outcome desirability (Niv, 2019; Wilson et al., 2014; Rudebeck et al., 2017),

70 and the lateral OFC may signal resource availability (Rudebeck et al., 2017). However, these

71 distinctions are based on coarse parcellation, and may not reflect the subtleties of anatomical and

72 functional differentiation within this broad swath of cortex.

73 Economic choice requires the transformation of sensory and mnemonic information into

74 actions (Cai \& Padoa-Schioppa, 2014; Hare et al., 2011; Hayden \& Moreno-Bote, 2018; Yim et

75 al., 2019; Yoo et al., 2018). In other words, economic choice involves a transformation from an

76 abstract (goods) space to a concrete (action) one (Padoa-Schioppa, 2011; Rangel et al., 2008). It

77 is likely that the OFC plays a central role in this process. However, the nature of that role remains

78 unclear. Many studies have emphasized the abstract side of OFC processing; however, a growing

79 number of studies suggest that it may have an important spatial role as well (Yoo et al., 2018; Luk

80 and Wallis, 2013; Strait et al., 2016; Roesch et al., 2006). The inconsistency across studies, along

81 with the functional divisions explained above, raise the possibility that different parts of OFC 
82 may have heterogeneous functions. Defining, and working with, that heterogeneity may allow for

83 more precise delineation of OFC function.

84 We hypothesized that the key to understanding the role of OFC in the transformation

85 from abstract to concrete representations is through its connectivity with another region involved

86 in economic choice: the posterior cingulate cortex (PCC). This region, located in the

87 posteromedial cortex, has not received the same amount of scholarly scrutiny from decision

88 neuroscientists as OFC. Nevertheless, the PCC has a confirmed spatial repertoire (Dean \& Platt,

89 2006; Hayden et al., 2008; Olson et al., 1996; Spreng et al., 2009; Dean et al., 2004) and plays a

90 central economic role (Barack et al., 2017; Hayden et al., 2008; Heilbronner \& Platt, 2013; Kable

91 \& Glimcher, 2007; Pearson et al., 2009; Young \& Mccoy, 2015). That is, while PCC has

92 consistent responses to outcomes, those responses are spatially selective, perhaps due to the

93 strong interactions between this region and the parietal cortex (Morecraft et al., 2004; Cavada et

94 al., 1989; Pandya \& Seltzer 1982). Finally, PCC has direct bidirectional communication with

95 OFC (Kobayashi \& Amaral, 2003; Morecraft et al., 2004; Pandya et al., 1981; Parvizi et al.,

96 2006; Morecraft et al., 1992). We wanted to probe how the OFC-PCC circuit might facilitate

97 transformations from abstract space to action space for choice.

98 


\section{RESULTS}

\section{OFC-PCC anatomical connectivity}

101 We injected the tracer fluororuby in the PCC gyrus, centered at the border between area

102 23a and 30 (with some involvement of area 29, Paxinos et al., 2009). This injection resulted in

103 widespread retrograde and anterograde labeling throughout the anterior and posterior cingulate

104 cortices, parietal lobe (precuneus and intraparietal sulcus), medial temporal lobe (hippocampal

105 formation), and frontal cortex (primarily dorsolateral prefrontal and orbitofrontal cortices).

106 Projections to the OFC were particularly interesting for their specificity: cells and terminal fields

107 were clustered around the medial orbital sulcus (mainly area 13a, but also including lateral 140

108 and caudal 11, based on Paxinos et al., 2009; Figure 1A-C). There were projections to other OFC

109 subregions, but these were noticeably less dense. These results are consistent with other, similarly

110 placed cases from the literature (Kobayashi \& Amaral, 2003; Morecraft et al., 2004; Pandya et al.,

111 1981; Parvizi et al., 2006; Morecraft et al., 1992). A second injection (Supplementary Figure 1)

112 targeted the PCC sulcus, and also resulted in labeling around the medial orbital sulcus, although it

113 was less specific. We concluded that although the PCC does connect with other OFC subareas

114 (OFCout), its relationship with the subareas surrounding the medial orbital sulcus (from here on

115 referred to as OFCin) is unique. We next sought to examine the functional properties of this

116 circuit. 

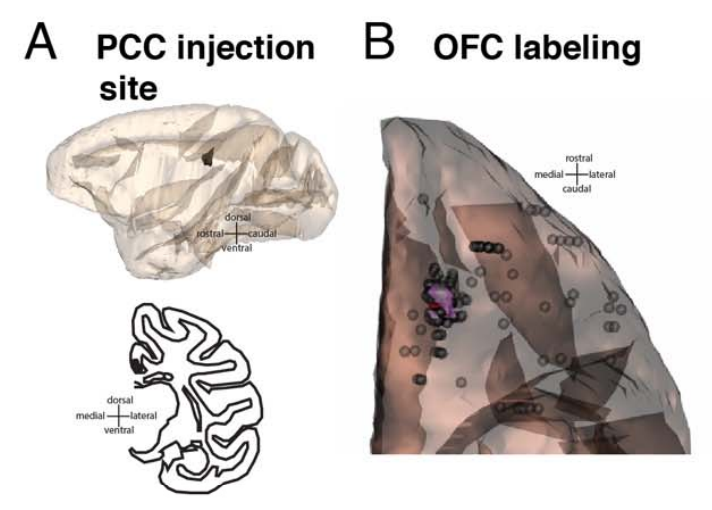

\section{OFC labeling}
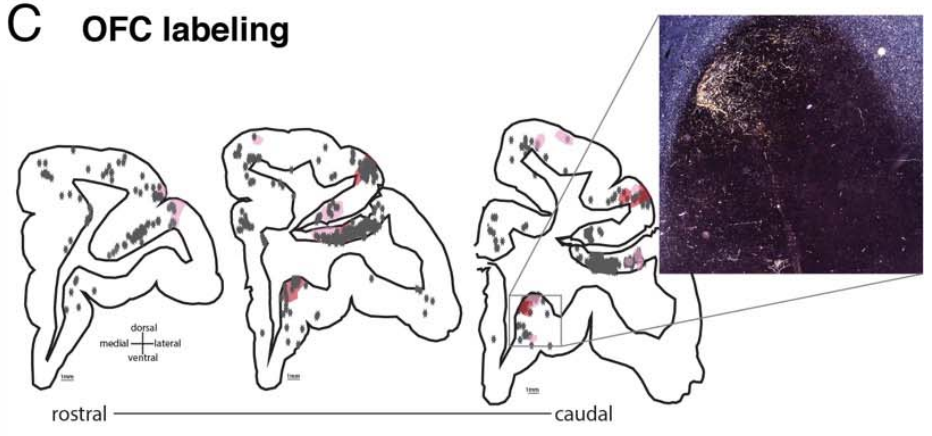

\section{D recording sites}

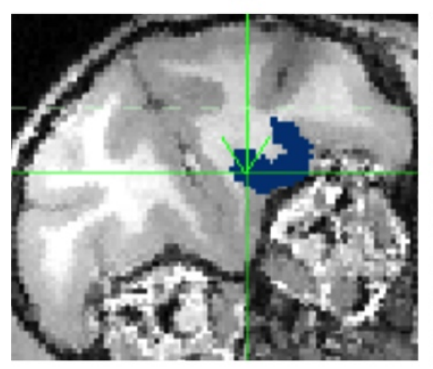

OFCin

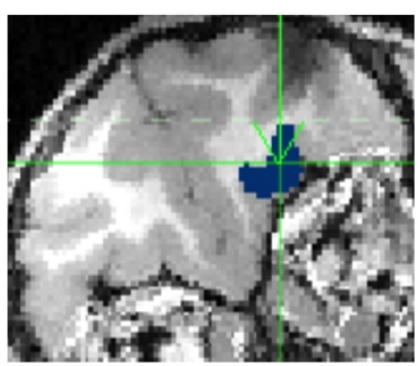

OFCout

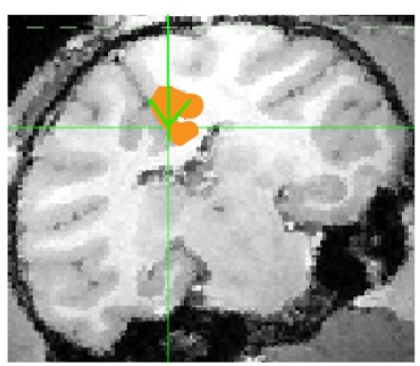

PCC
118

Figure 1. Anatomical connectivity between OFC and PCC and matching recording sites. A. Injection site (black, Case M1FR) is rendered in 3D and shown in a sagittal view (top) and on a coronal slice (bottom). B. Projections to the OFC rendered in 3D and shown on an orbital view. Red indicates dense terminal fields; pink indicates light terminal fields; gray spheres are labeled cells. The majority of OFC labeling is around the medial orbital sulcus. C. Coronal slices with full PFC labeling, colors are as in (B). A photomicrograph indicates label around the medial orbital sulcus. D. Coronal sections of example recording site from each of OFC (dark blue colored region), with OFCin on left and OFCout in the middle, and PCC (orange colored region).

\section{Behavior and electrophysiology}

We recorded neural activity in all three regions--PCC, OFCin, and OFCout (Figure 1D)-while rhesus macaques (Macaca mulatta, Subjects P and S) performed a well-established economic choice task (Strait et al., 2014; Farashahi et al., 2018; Figure 2A). The critical features of the task are its asynchronous presentation of options (offer 1 and offer 2) and the random order of presentation of options by location (left vs. right), which allowed us to examine the relationship between encodings of offer both abstractly (by time of presentation) and concretely 
137 (by side of presentation). As in our past studies using this task (e.g. Strait et al., 2014), both

138 subjects reliably chose the option with higher expected value, indicating high choice accuracy

139 (Supplementary Figure 2). We recorded neural ensembles with multiple linear probes

140 simultaneously in both PCC ( $\mathrm{n}=213$ neurons) and OFC ( $\mathrm{n}=98$ neurons, 44 in OFCin and 54 in

141 OFCout). 
A

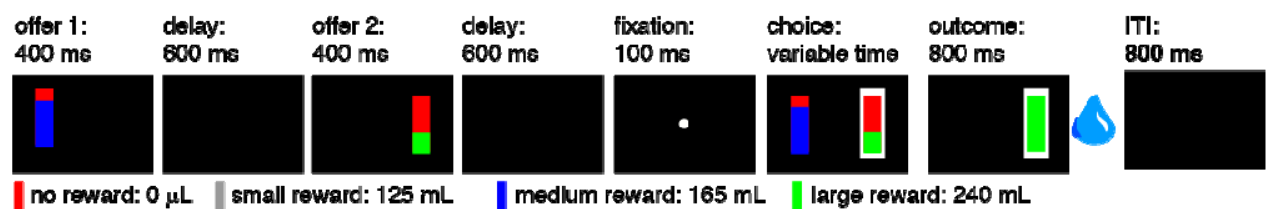

| no reward: $0 \mu \mathrm{L}$ || small reward: $125 \mathrm{~mL}$ | medlum reward: $165 \mathrm{~mL}$ | large reward: $240 \mathrm{~mL}$

B spike(OFCin) - field(PCC) coherence

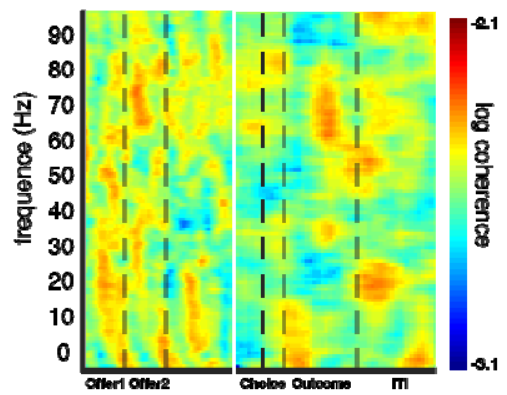

D

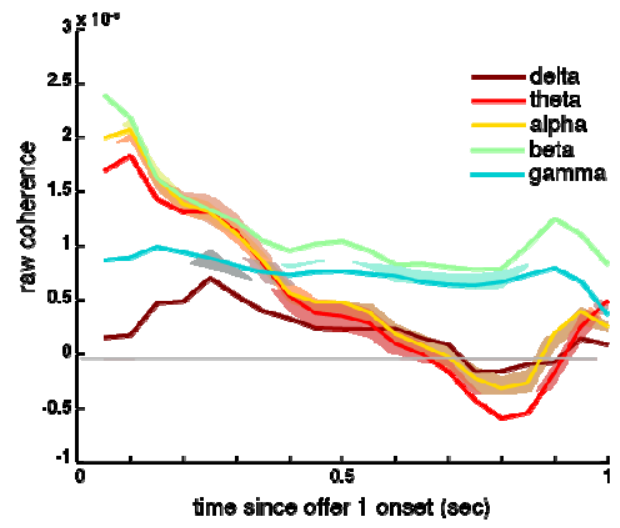

F

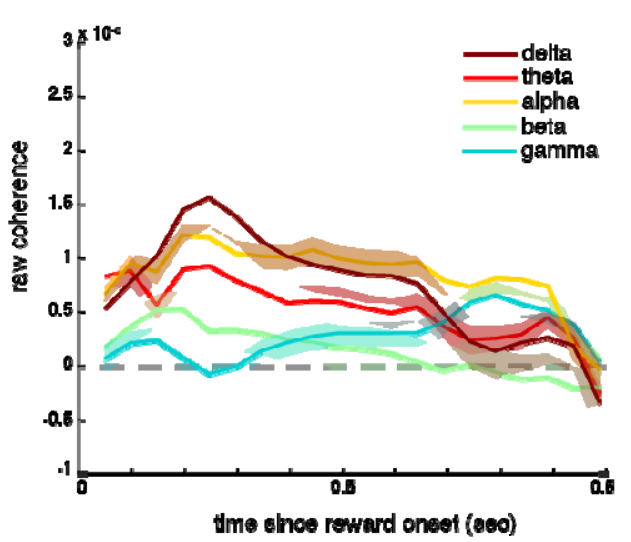

C spike(OFCout - field(PCC) coherence

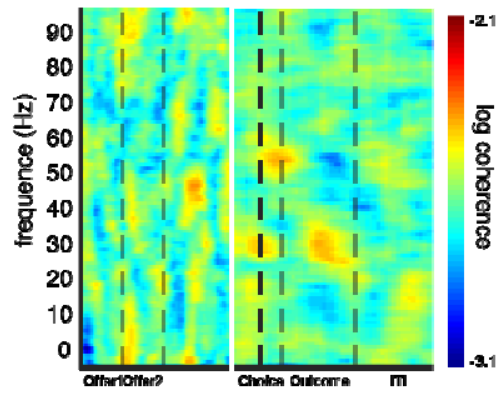

E

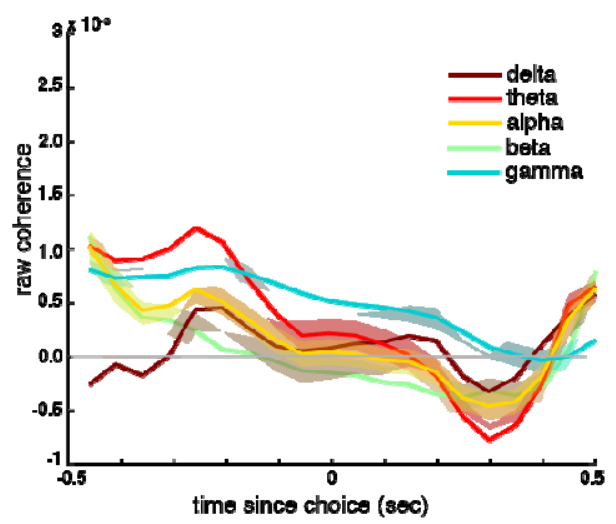

G

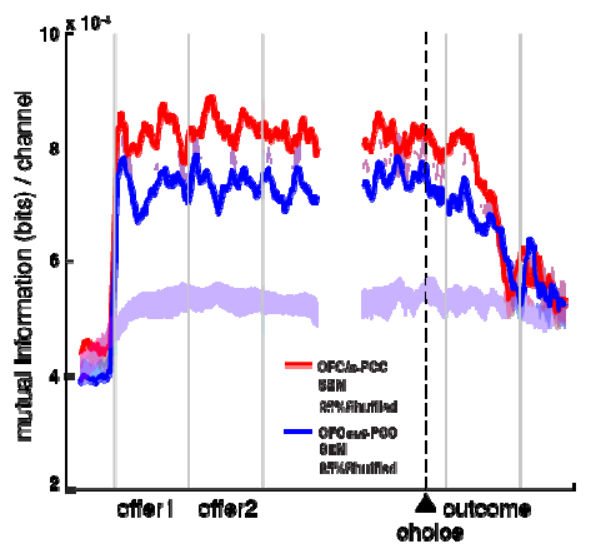

Figure 2. Task and functional connectivity. A. Two-option risky choice task. Black rectangles symbolize various task epochs subjects experience during task. Stakes are represented as different colors: small (gray), medium (blue), or large (green) reward. Losing the gamble (no reward) is represented in red. The height of the stakes-color region represents the probability of winning the gamble, and the height of the red-color 
region represents the probability of losing the gamble. The white frame around the right option in the choice epoch represents the scenario where the subject chooses the right option with eye fixation. The water droplet symbol indicates that reward delivery (or lack thereof) occurs. B. Trial-averaged spike-field coherence in OFCin spk $^{-} \mathrm{PCC}_{\text {lfp }}$ circuit. X axis: time in a trial. $Y$ axis: frequency. Color: strength of spike-field coherence on log10 scale (warmer colors=higher coherence). Data from the first half of the trial (offer period) was aligned at offer 1 onset. Data from the second half of the trial (choice period) was aligned at choice execution. C. Spike-field coherence in OFCout $t_{\text {spk }}-$ PCC $_{\text {lfp }}$. Conventions as in (B). D-F. Difference in spike-field coherence between the two circuits (coherence in OFC in spk $-\mathrm{PCC}_{\text {lfp }}$ circuit minus coherence in OFCout $t_{\mathrm{spk}}-\mathrm{PCC}_{\text {lfp }}$ circuit), broken down into different frequency bands as a function of time (Methods), during (C) offer 1 epoch, (D) choice epoch, and (E) reward epoch. G. Mutual information (averaged across number of channels) in OFCin-PCC and OFCout-PCC circuits. SEM: standard error of the mean. Red shaded area: SEM of mutual information in OFCin-PCC circuit. Blue shaded area: SEM of mutual information in OFCout-PCC circuit. Magenta and cyan shaded areas: the middle $95 \%$ range of the randomly shuffled mutual information (500 times) for OFCinPCC and OFCout-PCC circuits, respectively. Thus, the original (non-shuffled) mutual information values outside of the shaded area is significantly higher/lower than expected

\section{Functional connectivity}

We asked whether the OFCin-PCC circuit shows greater functional (rather than anatomical) connectivity than the OFCout-PCC circuit. We employed spike-field coherence,

171 which relates the recorded action potentials of one region to the local field potential (LFP)

172 oscillations of another (Buzsáki, 2004; Dal Monte et al., 2020; Buzsaki \& Draguhn, 2004;

173 Pesaran, 2010; Scherberger et al., 2005; Widge et al., 2019; see Methods and Supplementary

174 Figure 3). We found that broadband spike-field coherence between OFCin (spikes) and PCC

175 (LFPs) is stronger than coherence between OFCout and PCC. Specifically, during the offer

176 epoch, the broadband spike-field coherence in the OFCin ${ }_{\text {spk }}-\mathrm{PCC}_{\text {lfp }}$ circuit is higher than that in

177 the OFCout spk $_{1}-\mathrm{PCC}_{\text {lfp }}$ circuit $(\mathrm{z}=5.01, \mathrm{p}<0.001$, Wilcoxon signed rank test, Figure 2B-C). This

178 effect appears to be broadband; it is significant within all five bands that we tested: delta, theta,

179 alpha, beta, and gamma (Figure 2D). The same pattern occurs in the choice and outcome epochs

$180\left(\mathrm{OFCin}_{\text {spk }}-\mathrm{PCC}_{\text {lfp }}>\mathrm{OFC}_{\text {out }}\right.$ spk $_{\text {-PCC }}-\mathrm{PC}_{\mathrm{lf}}$; choice: $\mathrm{z}=2.81, \mathrm{p}=0.005$; outcome: $\left.\mathrm{z}=3.70, \mathrm{p}=0.005\right)$.

181 During choice, higher coherence occurs within the theta, alpha, and gamma bands, but not the

182 delta or beta bands (Figure 2E). During outcome, higher coherence occurs in all but the beta

183 band (Figure 2F). 
We next probed information exchange within our two newly identified circuits by

185 comparing mutual information within each one (see Methods). We computed channels as the set

186 of all possible pairs of trains from across the two regions (Timme \& Lapish, 2018). Thus, we

187 identified 9372 channels in the OFCin-PCC circuit and 11502 channels in the OFCout-PCC

188 circuit and calculated the averaged mutual information per channel within each circuit. We found

189 that the OFCin-PCC circuit shares higher mutual information than OFCout-PCC $\left(7.44 \times 10^{-4}\right.$ vs.

$1906.72 \times 10^{-4}$ bits/channel; $\mathrm{z}=17.47, \mathrm{p}<0.001$, Wilcoxon signed rank test). Mutual information in both

191 circuits increased significantly at task onset $(\mathrm{p}<0.025$, shuffle test, see Supplement), suggesting

192 that the observed mutual information effect reflects task-driven, rather than spontaneous,

193 fluctuations (Figure 2G).

\section{Neural computation}

Functional connectivity results do not speak to the content of the information transmitted.

197 We therefore analyzed encoding of task variables with a multiple linear regression model. All

198 three regions encoded offer and outcome values in their respective epochs with similar

199 proportions of neurons, encoding strengths, and latency (Supplement). They also all encoded the

200 chosen option (offer 1 vs. 2) and chosen location (left vs. right). However, OFCin encoded the

201 chosen option (offer 1 vs 2 ) with shorter latency ( 90 ms, F=3.35, p=0.037, GLM Gamma

202 distribution; Methods) than both OFCout (170 ms, $\mathrm{t}=-2.14, \mathrm{p}=0.033)$ and PCC (150 ms, $\mathrm{t}=-2.36$,

$203 \mathrm{p}=0.019$ ), suggesting chosen option information arises first in OFCin. PCC appears to be more

204 spatially sensitive than either OFC region: it showed a higher proportion of neurons encoding

205 chosen location than chosen option $\left(\chi^{2}=5.31, \mathrm{p}=0.021\right.$, chi-square test $)$; neither $\mathrm{OFC}$ region

206 shows this pattern (Supplement). PCC and OFCin also encoded the chosen location with

207 significantly shorter latencies than $\operatorname{OFCout~}(\mathrm{F}=5.71, \mathrm{p}=0.004$; Supplement), suggesting that PCC

208 and OFCin, but not OFCout, negotiate chosen location encoding. 
210 when offer 2 was revealed, a putative neural signature of value comparison (Azab \& Hayden,

211 2017; Strait et al., 2014). We performed this analysis using a 200-ms analysis window (350 ms

212 after offer 2 onset; the same window identified by the Granger analysis, see below) and found

213 that OFCin showed this putative mutual inhibition signal ( $\mathrm{r}=-0.36, \mathrm{p}=0.016$, Spearman

214 correlation; Figure 3A). We did not observe such an effect in OFCout $(\mathrm{r}=-0.18, \mathrm{p}=0.190$; Figure

215 3B $)$ or in PCC ( $r=0.02, p=0.943$; Figure 3C). We also did not find this negative correlation

216 during the later choice epoch (from $400 \mathrm{~ms}$ to $200 \mathrm{~ms}$ before choice action) in any of the three

217 regions (Supplementary Figure 4A-C). The effect size of these negative correlations was not

218 significantly different in OFCin vs. OFCout ( $\mathrm{z}=-0.93, \mathrm{p}=0.176$; Fisher's Transformation test) but

219 was significantly larger in OFCin than in PCC $(\mathrm{z}=-2.32, \mathrm{p}=0.010)$. These results suggest that both

220 OFC subregions, moreso than PCC, were involved in value comparison between offer 1 vs. offer

2212 in a presentation order frame, although the effect did not reach significance in OFCout alone.

To gain insight into the question of how effector-independent (order-based) value signals

223 are transformed into effector-dependent (spatial-based) ones, we next asked whether regression

224 coefficients for left and right offer values (EVl and EV $r$; as opposed to first and second as in the

225 previous analysis) were negatively correlated. In other words, relaying the mutual inhibition

226 signal to this framework would indicate that neurons carry a decision variable that could

227 potentially be read out by downstream motor areas to guide actions. We found this negative

228 correlation between $\mathrm{EV} l$ and $\mathrm{EV} r$ during the offer 2 epoch in $\mathrm{PCC}(\mathrm{r}=-0.24, \mathrm{p}<0.001$; Figure 3F),

229 but not significantly in OFCin $(r=-0.16, p=0.293$; Figure 3D) or OFCout $(r=0.10, p=0.454$,

230 Figure 3E). Interestingly, the effect size of these negative correlations was not significantly

231 different in OFCin vs. PCC ( $\mathrm{z}=-0.49, \mathrm{p}=0.313)$ but was significantly larger in PCC than in

232 OFCout $(\mathrm{z}=-2.21, \mathrm{p}=0.014)$. During the later choice epoch, we found the same signal in both PCC

$233(\mathrm{r}=-0.19, \mathrm{p}=0.006)$ and $\mathrm{OFC}$ in $(\mathrm{r}=-0.33, \mathrm{p}=0.029)$, but not $\mathrm{OFC}$ out $(\mathrm{r}=0.31, \mathrm{p}=0.022)$ 
234 (Supplementary Figure 4D-F). These results suggest that PCC and OFCin, but not OFCout,

235 were involved in value comparison between left vs. right offers.

To help understand whether the OFCin-PCC circuit transforms the mutual inhibition

237 signals from a value-based comparison to an action-based comparison, we measured Granger

238 causality between time series of mutual signals in OFCin and PCC calculated with a 200-ms

239 sliding window (see Methods). We found that the strength of mutual inhibition for EV1-EV2 in

240 OFCin Granger-caused the strength of mutual inhibition for $\mathrm{EV} l$-EV $r$ in $\mathrm{PCC}$ (gc=40.56,

$241 \mathrm{p}=0.019)$, with a $240 \mathrm{~ms}(4.17 \mathrm{~Hz})$ lag. In the reverse direction, the strength of mutual inhibition

242 for EVl-EVr in PCC Granger-caused the strength of mutual inhibition for EV1-EV2 in OFCin

$243(\mathrm{gc}=59.75, \mathrm{p}=0.014)$, but with a much longer lag $(380 \mathrm{~ms} ; 2.63 \mathrm{~Hz})$. In contrast, the strength

244 of mutual inhibition for EV1-EV2 in OFCout did not Granger-cause the strength of mutual

245 inhibition for EVl-EVr in PCC with any time lag (see Methods for controls for confounding

246 variables). These results suggest that through the communication in the OFCin-PCC circuit, but

247 not the OFCout-PCC circuit, the computation for value comparison transformed from value space

248 (in OFCin) to action space (in PCC).

249 If the previous result holds, then we would expect to decode choice signal more strongly

250 in value space (in the format of chosen option, offer 1 vs. 2) in OFCin but decode choice more

251 strongly in action space (in the format of chosen location, L vs. R) in PCC. We tested for this

252 using Linear Discriminant Analysis (LDA). Although chosen options (offer 1 vs. 2), chosen

253 location (left vs. right), and EV1 (high vs. low) were all significantly decodable from all three

254 regions, PCC indeed showed a significantly higher decodability for chosen location $\left(\chi^{2}=8.12\right.$,

$255 \mathrm{p}=0.004$; Figure 3G-H; Supplementary Figure 5). More importantly, we found that the

256 decodability for chosen option (offer 1 vs. 2) in OFCin Granger-caused the decodability for

257 chosen location (left vs. right) in PCC ( $\mathrm{gc}=11.19, \mathrm{p}=0.025)$ with a $200 \mathrm{~ms}(5 \mathrm{~Hz})$ lag. This

258 Granger-causal relation was absent on error trials $(\mathrm{gc}=3.04, \mathrm{p}=0.552)$. In the reverse direction, the

259 decodability for chosen location (left vs. right) in PCC Granger-caused the decodability for 
$261 \mathrm{~Hz}$ ). In contrast, the decodability for chosen option (offer 1 vs. 2) in OFCout did not Granger-

262 cause the decodability for chosen location (left vs. right) in PCC at any time lag (see Methods for

263 controls for confounding variables). These results suggest that the OFCin-PCC circuit, but not the

264 OFCout-PCC circuit, mediates the transformation of choice readout from a value-based to an

265 action-based framework. Speculatively, this transformation may be important for correct choice

266 behavior, since the both the decodability for choice and the Granger causal relation between

267 OFCin and PCC was disrupted in error trials (Supplement).
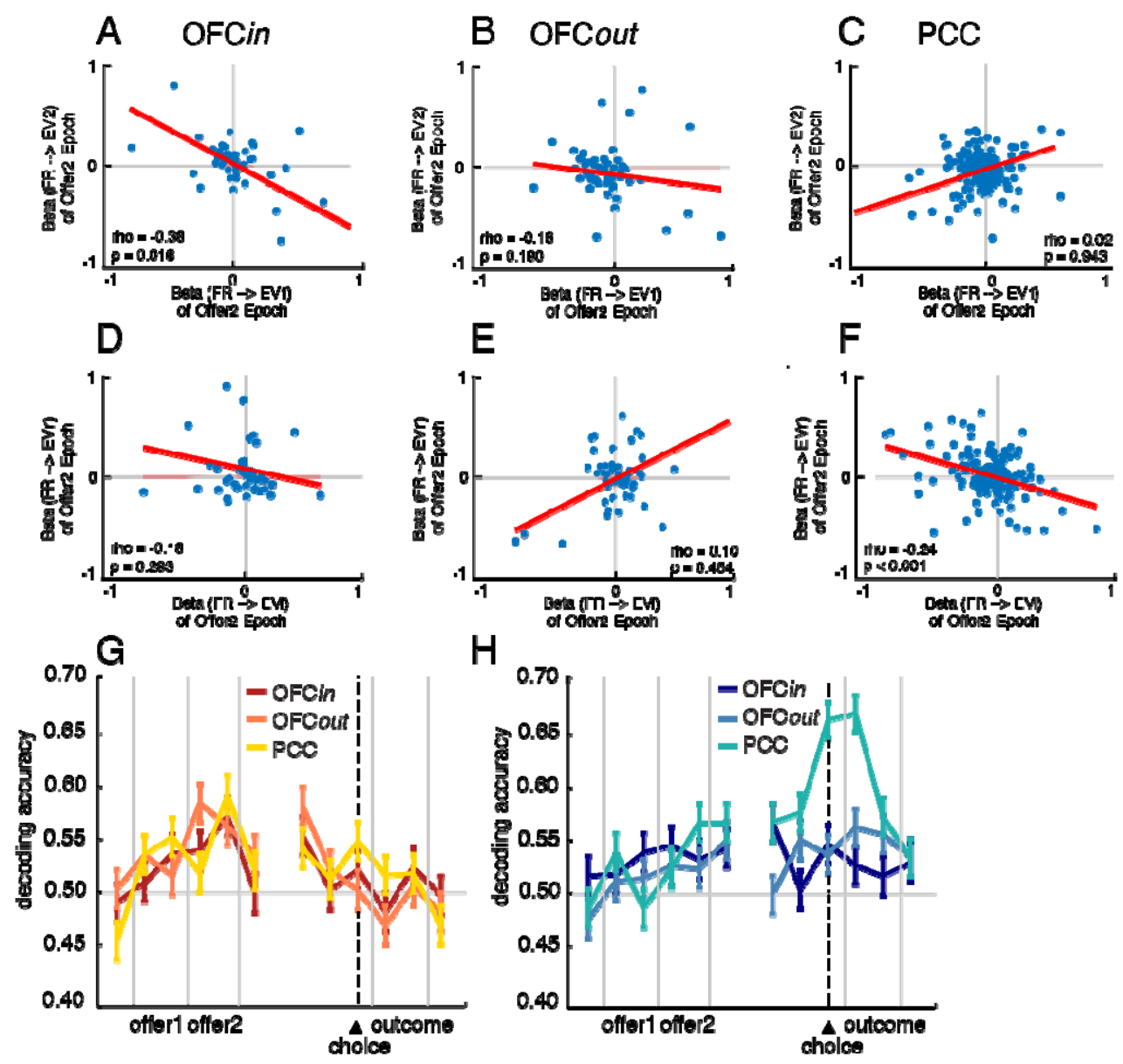

Figure 3. Neural computations. A-F: Scatter plots demonstrating population spreads for regression coefficients. Each dot represents one neuron; abscissa and ordinate represent regression coefficients for distinct (and uncorrelated) regressions. Shaded area: 95\% confidence interval. A-C: Putative mutual inhibition effects (Strait et al., 2014).

273 $Y$-axis indicates regression coefficient for expected value of offer 2 regressed against

274 firing rate in epoch 2. X-axis indicates regression coefficient for expected value of offer 1 
against firing rate in epoch 2. D-F: Putative mutual inhibition effects for space (new right offer against firing rate in epoch 2. X-axis: regression coefficient for expected value of left offer against firing rate in epoch 2. A,D: OFCin. B,E: OFCout. C,F: PCC. G-H: Decoding accuracy of choice ( $G$ is accuracy for offer 1 vs offer 2; $\mathrm{H}$ is accuracy for left vs right) based on firing rates using linear discriminant analysis. Y-axis: probability of decoding correctly. X-axis: time in a trial. Error bar: standard error of the mean.

284 Averbeck, 2020; Churchland et al., 2012; Mante et al., 2013; Yoo and Hayden, 2020) also reflect

285 the translation of choice from value space to action space in the OFCin-PCC circuit. Research in

286 motor generation has found that population activity dynamics in the premotor area during the

287 preparatory period determined the possible range of neural dynamics in the primary motor area

288 (M1), and this range determined what hand motion can be generated in M1 (Afshar et al., 2011).

289 To test whether this dynamical generative process of local neural computation could help explain

290 the relayed choice dynamics from abstract value space in OFCin to concrete action space in PCC,

291 we conducted PCA on trial-averaged population states for each region and then projected the

292 trial-averaged population activity onto the top-N principal component (PC) space that

293 cumulatively explained > 70\% of the variance (Methods; we developed this approach in Wang \&

294 Hayden, 2017). The projected population trajectories reflect the generative temporal evolution of

295 population dynamics (Figure 4A-F), and the separation between trajectories, which distinguished

296 task parameters, became significantly higher than shuffled chance level as the trial unfolded

297 (bottom shaded area). These distinctions diminished in error trials (Supplementary Figure 6),

298 suggesting that the population dynamics and their separation are indeed crucial for generating

299 correct choice behavior.

300 We then projected the trial-by-trial population states onto this top-N PC space to obtain

301 trial-by-trial population trajectories and used adjusted distance to measure the trajectory

302 separation (Methods; Murray et al., 2017). We found significantly larger trajectory separation for

303 chosen option (offer 1 vs. 2 ) in OFCin $\left(\chi^{2}=11.51\right.$, p=0.003, Kruskal-Wallis test with Tukey- 
304 Kramer multiple comparison) than in OFCout (OFCin>OFCout: $\mathrm{p}=0.007$ ) and PCC

305 (OFCin>PCC: $\mathrm{p}=0.012$; no significant difference between OFCout and PCC, $\mathrm{p}=0.988$ ). This

306 result highlights the specific role of OFCin in mediating abstract comparison.

307 In contrast, we found significantly larger trajectory separation for chosen location (left vs

308 right $)$ in PCC $\left(\chi^{2}=6.27, p=0.043\right.$, Kruskal-Wallis test with Tukey-Kramer multiple comparison)

309 than in OFCout (PCC>OFCout: $\mathrm{p}=0.043$ ) but not in OFCin (PCC $\approx$ OFCin: $\mathrm{p}=0.829$; there was no

310 significant difference between OFCin and OFCout, $\mathrm{p}=0.164)$. There was also no such cross-

311 region distinction for EV1 (high vs. low; Supplement; Supplementary Figure 6). The trajectory

312 separation differences for chosen option and chosen location were also absent in error trials

313 (Supplement), consistent with the intuitive idea that the areal difference in the unfolding

314 trajectory separation contributes to correct choice behavior.

315 The separation between population trajectories for chosen option (offer 1 vs 2) in OFCin

316 Granger-caused the separation between population trajectories for chosen location (left vs. right)

317 in PCC ( $g c=9.98, p=0.019)$, with a $150 \mathrm{~ms}(6.67 \mathrm{~Hz})$ lag. In the reverse direction, the distance

318 between population trajectories for chosen location (left vs. right) in PCC Granger-caused the

319 distance between population trajectories for chosen option (offer 1 vs. 2) in OFCin ( $\mathrm{gc}=17.28$,

$320 \mathrm{p}=0.016)$ but with a much longer lag $(350 \mathrm{~ms} ; 2.86 \mathrm{~Hz})$. Interestingly, this "feedback" influence

321 seems to amplify the OFCin to PCC input $300 \mathrm{~ms}$ after the first instance of Granger causal

322 influence, by increasing the Granger-causality from OFCin to PCC ( $g c=38.29, \mathrm{p}<0.001$;

$323 \mathrm{lag}=450 \mathrm{~ms} ; 2.22 \mathrm{~Hz}$ ). In contrast, the distance between population trajectories for chosen option

324 (offer 1 vs. 2) in OFCout did not Granger-cause the distance between population trajectories for

325 chosen location (left vs. right) in PCC with any time lag (see Methods for the control for

326 confounding variables). These results suggest that while local neural computation was generating

327 choice representations, their unfolding population dynamics also interact with the generative

328 dynamics in other regions. We found the dynamics in OFCin-PCC, but not those in OFCout-PCC, 
329 morphed from developing the separation for choice in value space to developing the separation

330 for choice in action space.

331

A OFCin
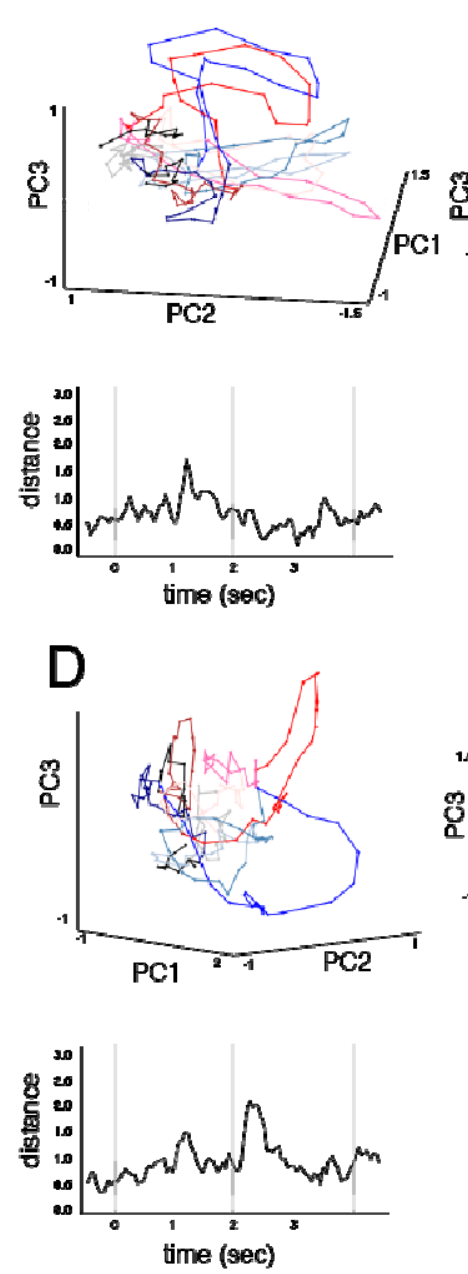

\section{G}

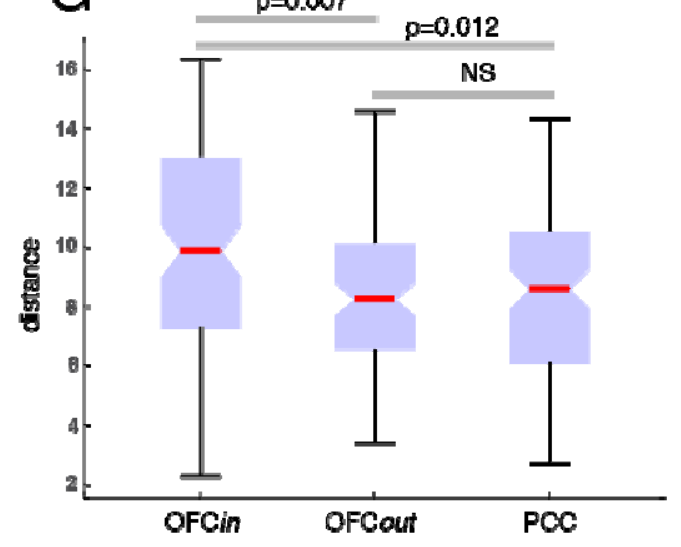

B OFCout

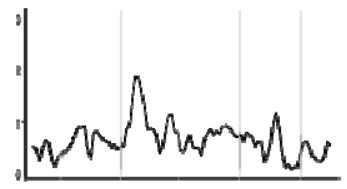

$\mathrm{E}$
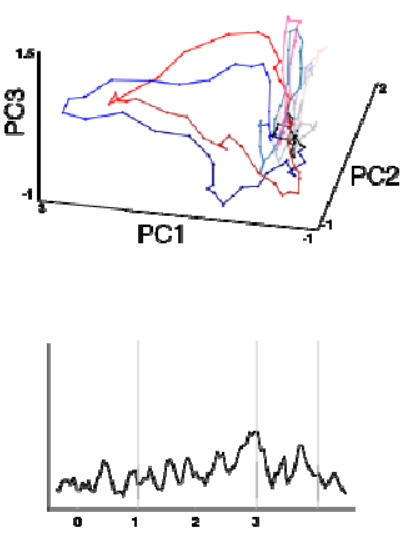
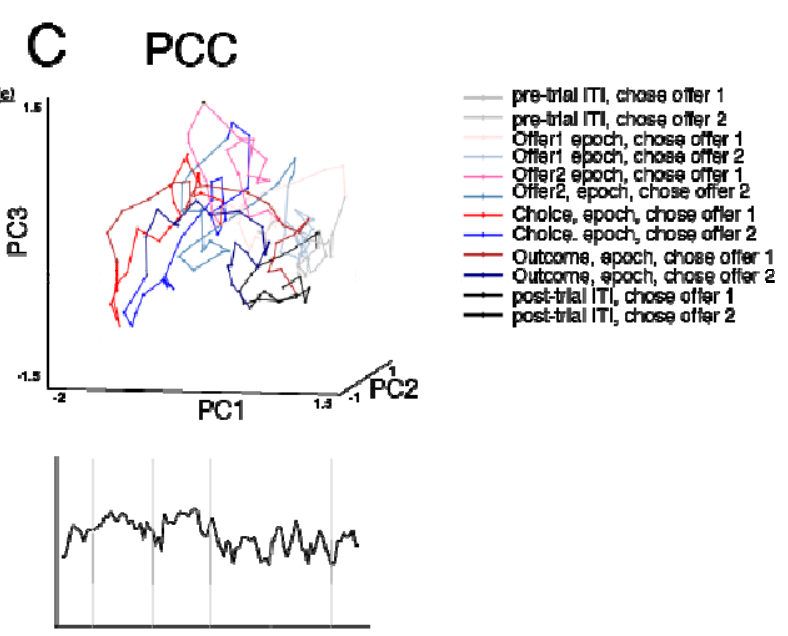

F
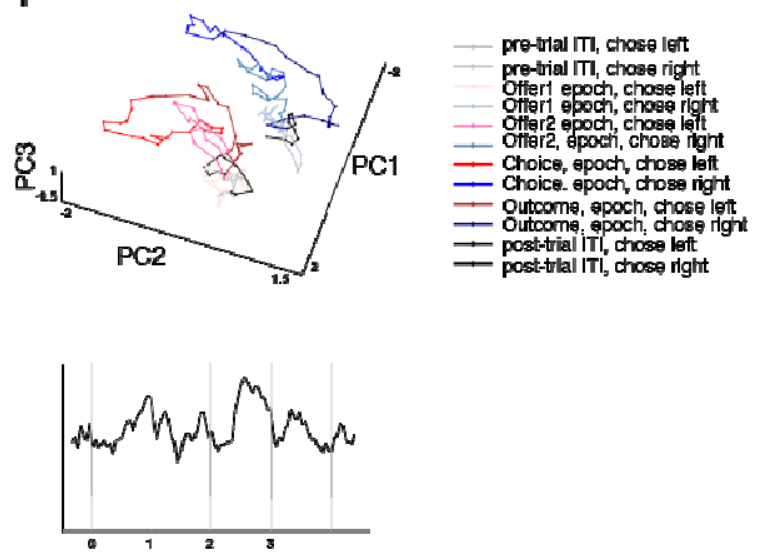

332

$\mathrm{H}$

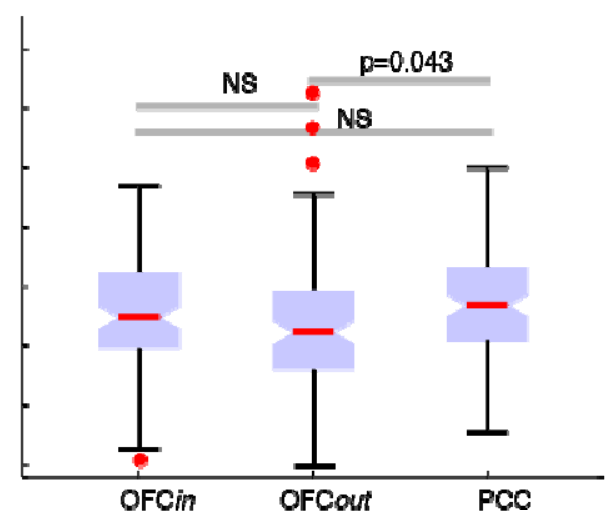


333 Figure 4: Top plots: trial averaged population activity projected onto top-N PC space (only top-3

334 PCs are shown here), separated by choice option (offer 1 vs 2) (A-C) or choice location (D-F), in OFCin (left column), OFCout (middle column), and PCC (right column). Warm colors: trial averaged population activity for choosing offer $1(\mathbf{A}-\mathbf{C})$ or left offer $(\mathbf{D}-\mathbf{F})$. Cool colors: trial averaged population activity for choosing offer 2 (a-c) or right offer (d-f). Colors indicate each of the following epochs: the ITI before the current trial, the offer 1 epoch, offer 2 epoch, choice epoch, outcome epoch, and the ITI after the current trial. Bottom plots: separation measured by Euclidean distance between averaged population trajectories (warm and cool colored lines). Yaxis: Euclidean distance. X-axis: time in a trial. Dark line: distance between trial-averaged trajectories for choosing offer 1 vs. offer 2 (A-C) or choosing left vs. right offer (D-F). Shaded area: middle 95\% trial-averaged Euclidean distance between population trajectories from condition-shuffled data. Shuffle was only based on the choice of offer 1 or offer 2 (A-C) or on the choice of left or right offer (D-F), the cell identities and temporal orders were not shuffled. Euclidean distance (i.e. separation; dark line) beyond the shaded area is significant $(p<0.05)$. Specifically, the distance (dark line) larger than (above) the shaded area is where separation between population trajectories is significantly larger than expected by chance $(p<0.025)$. These significant portions mark when the population activity dynamics significantly reflected the choice of offer 1 or offer 2 (A-C) or on the choice of left or right offer (D-F). G-H: Bottom: ranked trialby-trial adjusted distance. Kruskal-Wallis box plot. The red horizontal line: the median. The bottom and top edges of the box: the 25th and 75th percentiles. The whiskers extend to the most extreme data points not considered outliers. ' + ' individual outliers. 


\section{DISCUSSION}

Here we report the existence of two functionally distinct subregions within the OFC that

357 can be differentiated by their connectivity with the PCC, both anatomically and functionally.

358 OFCin, located on the banks of the medial orbital sulcus, seems to have stronger anatomical

359 connectivity with PCC than OFCout, which for our electrophysiological recordings was situated

360 lateral to OFCin. This boundary seems to correspond to a functional separation that relates to the

361 negotiation between abstract (goods-based) and motor (action-based) modalities. The abstraction

362 transformation is mediated by an OFCin-PCC circuit (that is anatomically and functionally

363 connected) and is uncoverable using analyses of value comparison, decoding, and population

364 dynamics. Crucially, instead of copying the more abstract choice signal from OFCin, computation

365 within PCC (perhaps with assistance from other input structures) adopts a spatial framework,

366 which allows it to compare and represent the choice in a more concrete, action-based manner.

367 This influence is also bidirectional, with a later PCC (possibly feedback) influence that relays

368 choice from action space back to value space in OFCin, and OFCin in turn exerts an even

369 stronger influence of relaying choice from value space again to action space in PCC.

370 We speculate that OFCin-PCC forms a bidirectional, mutually excitatory circuit. Our data

371 support the hypothesis that within both regions, a mutually inhibitory local circuit exists to

372 compare offers - in value space in OFCin but in action space in PCC. This circuit potentially

373 locks its computation with theta and delta band oscillations to translate choice representation

374 from abstract value space to concrete action space. Moreover, we did not see the information

375 relay between OFCin and PCC in error trials, suggesting that the transformation of choice in the

376 OFCin-PCC circuit is essential for generating a correct choice. Presumably, after the relay of

377 information between OFCin and PCC, a downstream area could use the action-bounded choice

378 signal to form an action plan.

379 Searching for reward signals in the brain leads to an embarrassment of riches (Vickery et al.,

380 2011; Rushworth et al, 2011; O'Doherty, 2014). The abundance of value is itself mysterious - why would 
381 the brain have so many seemingly redundant signals? One possibility is that different value correlates

382 have subtly different roles. That is, they may help negotiate the transform from abstract to concrete spaces

383 in different ways. Our results point to one possible case of this distinction, where some OFC value signals

384 are relatively abstract and others are relatively concrete, but the concrete (motoric) aspects of OFC signals

385 are derived from more specialized PCC signals. More speculatively, our results suggest that even apparent

386 intra-areal redundancy of function may mask an underlying heterogeneity of function. 


\section{METHODS}

\section{$390 \quad$ Neuroanatomy studies}

We injected the bidirectional tracer fluororuby into the PCC of two adult male rhesus macaque

392 (Macaca mulatta) subjects. In one (M1FR), the injection site was located at the border of areas 23 and 30

393 (with some involvement of area 29). In another (M6FR), the injection site was located at the border of

394 areas 23 and 31. We note that, although the PCC is often defined as areas 23 and 31, with areas 29 and 30

395 instead defined as retrosplenial cortex (Vogt et al., 2006; Leech et al., 2011), we were interested in the

396 functionality of this entire caudal cingulate region. Thus, like some prior authors (Armstrong et al., 1986;

397 Zilles et al., 1986; Mitelman et al., 2005; Vogt et al., 1992), here we defined PCC as areas 23, 31, 29, and 39830.

Prior to surgery, anatomical T1 and T2-weighted MRIs (3T for M1FR and 10.5T for M6FR) were

400 obtained at University of Minnesota's Center for Magnetic Resonance Research. Stereotaxic earbars were

401 filled with Vitamin E solution to visualize on the MRI and guide tracer placement relative to stereotaxic

402 zero.

On the day of surgery, monkeys were tranquilized by intramusculuar injections of ketamine

$404(10 \mathrm{mg} / \mathrm{kg})$, midazolam $(0.25 \mathrm{mg} / \mathrm{kg})$ and atropine $(0.04 \mathrm{mg} / \mathrm{kg})$. A surgical plane of anesthesia was then

405 maintained via the administration of inhalation of isofluorane (1-3\%). Monkeys were placed in a

406 stereotaxic instrument (Kopf Instruments), a midline scalp incision was made, and the muscle and fascia

407 were displaced laterally to expose the skull. A craniotomy $\left(\sim 2-3 \mathrm{~cm}^{2}\right)$ was made over the PCC, and small

408 dural incisions were made only at injection sites. Both monkeys received injections of FR (50nl, 10\% in

409 0.1M PB, pH 7.4, Invitrogen) in the PCC, as well as injections of additional tracers (lucifer yellow,

410 fluorescein, wheat germ agglutinin conjugated to horseradish peroxidase) in other regions not described

411 here. These do not cross-react with FR and were made distant from the PCC site. Tracers were pressure-

412 injected over 10 min using a $0.5-\mu l$ Hamilton syringe. Following each injection, the syringe remained in

413 situ for 20-30 min. Twelve to 14 days after surgery, monkeys were again deeply anesthetized and

414 perfused with $4 \mathrm{~L}$ of saline followed by $6 \mathrm{~L}$ of a $4 \%$ paraformaldehyde/1.5\% sucrose solution in $0.1 \mathrm{M} \mathrm{PB}$, 
$415 \mathrm{pH}$ 7.4. Brains were postfixed overnight and cryoprotected in increasing gradients of sucrose (10, 20, and

$41630 \%$ ). Serial sections of $50 \mu \mathrm{m}$ were cut on a freezing microtome into cryoprotectant solution.

417 One in eight sections was processed free-floating for immunocytochemistry to visualize the

418 tracer. Tissue was incubated in primary anti-FR (1:6000; Invitrogen) in 10\% NGS and 0.3\% Triton X-100

419 (Sigma-Aldrich) in PO4 for 4 nights at $4^{\circ} \mathrm{C}$. After extensive rinsing, the tissue was incubated in

420 biotinylated secondary antibody followed by incubation with the avidin-biotin complex solution

421 (Vectastain ABC kit, Vector Laboratories). Immunoreactivity was visualized using standard DAB

422 procedures. Staining was intensified by incubating the tissue for 5-15 s in a solution of $0.05 \%$ DAB

423 tetrahydrochloride, $0.025 \%$ cobalt chloride, $0.02 \%$ nickel ammonium sulfate, and $0.01 \% \mathrm{H} 2 \mathrm{O} 2$. Sections

424 were mounted onto gel-coated slides, dehydrated, defatted in xylene, and coverslipped with Permount.

425 Using a Zeiss M2 AxioImager, light microscopy was used to outline brain sections, PCC injection

426 sites, frontal cortical terminal fields, and frontal cortical labeled cells on 1 in 24 sections (1.2mm apart).

427 Terminal fields were outlined in darkfield using a 2.0, 4.0, or 10× objective with Neurolucida software

428 (MicroBrightField Bioscience). Terminal fields were considered dense when they could be visualized at a

429 low objective (2.6×) (Haber et al. 2006); otherwise, terminal fields were considered sparse. Thin, labeled

430 fibers containing boutons were marked as terminating; thick fibers without boutons were considered

431 passing. Retrogradely labeled cells were identified under brightfield microscopy (20x) using

432 StereoInvestigator software (MicoBrightField Bioscience).

433 Cases were registered and rendered in 3D in the following way. For each case, a stack of 2D

434 coronal sections was created from its Neurolucida chartings. This stack was imported into IMOD

435 (Boulder Laboratory for 3D Electron Microscopy, Kremer et al. 1996), and a 3D reconstruction that

436 contained the injection sites, terminal fields, and cells was created for each case separately. To render

437 these and merge cases together, we used a reference model from the NIMH Macaque Template (Seidlitz

438 et al., 2017), imported into IMOD. Placement of all contours-injection sites, terminal fields, cells, area

439 outlines-were assessed according to cortical and subcortical landmarks in the brain, then checked with

440 the original case and corrected as needed. 


\section{Neurophysiology studies}

Subjects. Two male rhesus macaques (Macaca mulatta) served as subjects to the

444 neurophysiology experiment. All animal procedures were approved by the University Committee

445 on Animal Resources at the University of Rochester (neurophysiology studies) and by the

446 Institutional Animal Care and Use Committee at the University of Minnesota (neurophysiology

447 and neuroanatomy studies). The experiments were designed and conducted in compliance with

448 the Public Health Service's Guide for the Care and Use of Animals. These subjects were used in

449 past studies involving set shifting and risky choice (Sleezer et al., 2016; Pirrone et al., 2018;

450 Heilbronner and Hayden, 2016).

453 central OFC and PCC (Paxinos et al., 2009; see also Öngür \& Price, 2000; Leech \& Sharp, 2014;

454 Mufson and Pandya, 1984; Figure 1D). Note that this posterior region is overlapping with but

455 ventral to a region we have previously recorded in known as CGp (Heilbronner et al., 2013;

456 Hayden et al., 2009; Hayden et al., 2010). Position was verified by magnetic resonance imaging

457 with the aid of a Brainsight system (Rogue Research Inc.) for subject P and Cicerone system (Dr.

458 Matthew D. Johnson at University of Minnesota) for subject S. Neuroimaging was performed at

459 the Rochester Center for Brain Imaging, on a Siemens 3T MAGNETOM Trio Tim using 0.5 mm

460 voxels. We confirmed recording locations by listening for characteristic sounds of white and gray

461 matter during recording, which in all cases matched the loci indicated by the Brainsight system or

462 Cicerone system.

465 using the same microdrive system until positioned within the OFC. Following a settling period,

466 all active cells were recorded. Electrodes were lowered using a microdrive (NAN Instruments) 
467 until the target region was reached. This lowering depth was predetermined and calculated with

468 the aid of either Brainsight or Cicerone system to make sure the majority of the contacts on the V-

469 probe were in the gray matter of the recording region. Individual action potentials were isolated

470 on a Ripple Grapevine system (Ripple, Inc.). Neurons were selected for study solely on the basis

471 of the quality of isolation; we never pre-selected based on task-related response properties. Cells

472 were sorted offline with Plexon Offline Sorter (Plexon, Inc.) by hand by MZW. No automated

473 sorting was used.

474

Eye Tracking and Reward Delivery. Eye position was sampled at 1,000 $\mathrm{Hz}$ by an infrared

476 eye-monitoring camera system (SR Research). Stimuli were controlled by a computer running

477 MATLAB (Mathworks) with Psychtoolbox (Brainard, 1997) and Eyelink (Cornelissen et al.,

478 2002) Toolbox. A standard solenoid valve controlled the duration of fluid reward delivery. For

479 part of the behavioral training, subjects received grape juice or cherry coke instead of water as

480 reward. However, water reward was used during all neural recording sessions. The relationship

481 between solenoid open time and water volume was established and confirmed before, during, and

482 after recording.

Behavioral task. Subjects performed a two-option gambling task identical to the one we used in a

485 previous investigation (Figure 1, Strait et al., 2014; see Heilbronner, 2017 for context). Two offers were

486 presented on each trial. Each offer was represented by a rectangle 300 pixels tall and 80 pixels wide

$487\left(11.35^{\circ}\right.$ of visual angle tall and $4.08^{\circ}$ of visual angle wide). Options offered either a gamble or a safe

488 (100\% probability) bet for liquid reward. Gamble offers were defined by both reward size and probability,

489 which were selected with uniform probabilities and independently of one another for each offer and for

490 each trial. Each gamble rectangle had two sections, one red and the other either blue or green. The size of

491 the blue or green portions indicated the probability of winning a medium (165 $\mu \mathrm{L})$ or large reward $(240$

$492 \mu \mathrm{L}$ ), respectively (Figure 1). These probabilities were drawn from a uniform distribution between $0 \%$ and 
$493100 \%$. Safe offers (1 out of every 8 offers) were entirely gray, and selecting one would result in a small

494 reward $(125 \mu \mathrm{L}) 100 \%$ of the time.

495 Offers were separated from the central fixation point by 550 pixels $\left(27.53^{\circ}\right.$ of visual angle). The

496 sides of the first and second offer (left or right) were randomized each trial. Each offer appeared for 400

497 ms followed by a $600 \mathrm{~ms}$ empty screen. After the offers were presented one at a time, a central fixation

498 point appeared, and the monkey fixated on it for $100 \mathrm{~ms}$. Then both offers appeared simultaneously and

499 the animal indicated its choice by shifting gaze to its preferred offer, maintaining fixation on it for 200

500 ms. Failure to maintain gaze for $200 \mathrm{~ms}$ would return the monkey to a choice state. Thus, subjects were in

501 theory free to change their mind if they did so within $200 \mathrm{~ms}$ (although they seldom did). Following a

502 successful 200-ms fixation, the gamble was immediately resolved and a liquid reward was delivered.

503 Trials that took more than $7 \mathrm{sec}$ were considered inattentive and were excluded from analysis (this

504 removed $<1 \%$ of trials). Outcomes that yielded rewards were accompanied by a white circle in the center

505 of the chosen offer (see Figure 1B). Each trial was followed by an 800-ms inter-trial interval (ITI) with a

506 blank screen.

507 Probabilities were drawn from uniform distributions with resolution only limited by the size of

508 the screen's pixels, which let us present hundreds of unique gambles. Offer reward sizes were selected at

509 random and independent of one another with a $43.75 \%$ probability of blue (medium reward) gamble, a

$51043.75 \%$ probability of green (large reward) gambles, and $12.5 \%$ probability of safe offers. Note that this

511 means two offers with the same reward size could be (and often were) presented in the same trial.

Statistical analyses: Behavior. Only trials accompanying the recording sessions were analyzed for

514 the current paper. For choice accuracy, we defined the correct choice as choosing the offer with expected

515 value higher than or equal to that of the alternative offer. Expected value (EV) is the product of stakes

516 multiplied by probability of winning (getting rewarded, in contrast to getting no reward). Probability of

517 choosing offer 1 as a function of value difference (EV1-EV2) is fitted with generalized linear with logistic 
518 transform function and binomial distribution. The error bars indicate $95 \%$ confidence intervals from the

519 logistic regression model.

522 recording sessions along with spike data using the Ripple Grapevine system. LFP data from each contact

523 of the Plexon v-probes were used. Raw data was low-pass filtered at $100 \mathrm{~Hz}$ and notch-filtered at $60 \mathrm{~Hz}$.

524 All filtering and frequency-domain (spectral) analyses were conducted in Matlab with Chronux toolbox

525 (Bokil et al., 2010). Power spectra in all three regions were calculated with all LFP channels. Spike-field

526 coherence was calculated using every combination of each spike train in one area and each channel of

527 LFP in another area. Coherence comparison used non-parametric statistics: Wilcoxon signed rank test and

528 Kruskal-Wallis test, both conducted in Matlab. We used the following bandwidths for analyses: Delta

529 (0.5-5 Hz), Theta (5-10 Hz), Alpha (10-15 Hz), Beta (15-30 Hz), and Gamma (>30 Hz). For coherence

530 comparisons, we calculated the coherence with a frequency-resolved method, such that we re-adjusted the

531 sliding calculation window widths to be four times the max length for each frequency band. We aligned

532 data to either offer 1 or choice to achieve a better temporal resolution of the coherence tests.

536 quantifies the information $X$ gives upon observing $Y$ and is the same as the information $Y$ gives upon

537 observing $X$. Equivalently, it captures how much uncertainty about $X$ decreases after learning $Y$, and vice

538 versa. We used the Neuroscience Information Theory Matlab toolbox to calculate the mutual information

539 between two spike trains, one from each brain area of interest (Timme \& Lapish, 2018).

540 To test whether the mutual information in OFCin-PCC or OFCout-PCC during task was higher

541 than expected chance, we shuffled each single-unit's brain area identity to form shuffled ensembles with

542 the same sizes as the original data. Then we shuffled temporal sequences within ITI and, separately,

543 within active task-time. The temporal shuffling is to test whether the increase in mutual information was 
544 above chance level and driven by engaging in the task. We then calculated mutual information based on

545 these shuffled ensembles. We repeated this procedure 500 times and obtained the middle $95 \%$ range of

546 the shuffled mutual information as a function of time (Figure 3F, shaded magenta and cyan for OFCin-

547 PCC and OFCout-PCC circuits, respectively). Thus, any value outside the shaded area is significantly

548 higher/lower than expected by chance.

Statistical analyses: Encoding. We used a sliding multiple linear regression to characterize the

551 encoding of all task variables (stakes, probabilities, expected values of offer 1 and offer 2, chosen option,

552 chosen location, whether offer 1 was presented on left vs. right, and choice outcome [win or lose * stakes

553 ]). To do so, we took the normalized firing rates (FR) of each neuron, averaged across a $200 \mathrm{~ms}$ time bin,

554 and then regressed against task parameters. The sliding procedure slid forward with a $10 \mathrm{~ms}$ step size. For

555 offer epochs, we used a multiple linear regression model with stakes, probabilities, and expected values

556 (EV) as predictors. Expected value is defined as the product of stake and probability. For the rest of the

557 epochs, we used a multiple linear regression model with stakes, probabilities, EV1, EV2, chosen option

558 (offer 1 vs. 2), chosen location (left vs. right), outcome (received outcome, 0 for lost gamble, reward of

559 the stake's size for won gamble), and whether offer 1 appeared on the left or right side of the screen. For

560 later tests looking the expected value tuning for left and right offers, we used a multiple linear regression

561 model with stakes, probabilities, left EV (EVl), right EV (EVr), chosen option (offer 1 vs. 2), chosen

562 location (left vs. right), outcome (actually received outcome, 0 for lost gamble, reward of the stake's size

563 for won gamble), and whether offer 1 (first appeared offer) appeared on the left or right side of the screen.

564 All predictors were centered and converted to categorical variables when applicable. The response

565 variable, firing rates, were normalized for each neuron across trials to avoid spurious correlation

566 (Blanchard and Hayden, 2014).

567 Proportion of neurons was calculated based on whether neurons significantly encoded a single

568 parameter of interest. Encoding strength was defined as the t-statistics of each predictor variable from the

569 multiple regression. We used t-statistics since they are not influenced by the actual range of each variable 
570 (even though we centered all predictor variables) and are comparable across variables. The comparison of

571 encoding strength across all three regions used the nonparametric Kruskal-Wallis test. Latency was

572 defined as, within the analyzed event window, the time lapsed until the encoding strength of the variable

573 of interest reached the peak for each neuron. Then the peak time for a region was calculated as the median

574 of each neuron's peak time. Latency calculation was based on all neurons and not only the significantly

575 tuned ones. Whether latencies from all three regions were significantly different from one another was

576 tested with generalized linear model (GLM) with a Gamma distribution, due to the fact that timing data,

577 such as latency or reaction time, are better described by a Gamma distribution than a Gaussian

578 distribution.

579 For mutual inhibition, we took the regression coefficients from the above described multiple

580 regression models for the offer 2 epoch and the choice epoch respectively. Then we correlated the

581 coefficients for offer 1 vs. 2 or EV $l$ vs. EV $r$ with a Spearman correlation. Spearman correlation is chosen

582 to avoid spurious correlation caused only by a few outliers. The strength of mutual inhibition signal is the

583 Spearman correlation coefficient.

Statistical analyses: Granger causality. Granger causality measures how one time series could predict (Granger-cause) another time series, after controlling for the fact that the later time series's early

587 sequences also predicts its own later sequences (Granger, 1969). Sometimes, calculation of Granger

588 causality is also conditioned on simultaneously observing other potentially confounding time series

589 (Lutkepohl, 2007). For all Granger causality tests, we first used the Augmented Dickey-Fuller test with

590 the autoregressive model with drift variant (ARD) to determine whether a time series was stationary.

591 Then we used the vector autoregression (VAR) model to determine the best time lag to use through model

592 comparison (Akaike information criterion) with different time lags. Then the Granger causality test was

593 used on stationary time series or with a correction for non-stationary time series. All Granger causality

594 analyses in this paper tested the Granger-causal relation between two key variables but also included the

595 conditional term with all other potentially confounding variables. That is, none of the potentially 
confounding decodability could explain the effect we saw. All Granger causality tests were carried out in Matlab. Matlab functions used: adftest, varm, estimate, summarize, gctest, the Econometrics Toolbox. testing of the linear discriminant analysis (LDA) decoder. For each trial, we aligned the normalized firing rates of each neuron at the onset of offer 1 presentation and took firing from $500 \mathrm{~ms}$ before this onset

602 through $2500 \mathrm{~ms}$ after this onset as the offer period (including $500 \mathrm{~ms}$ ITI before offer 1, offer 1 epoch,

603 offer 2 epoch, and the first $500 \mathrm{~ms}$ of decision-making). We also aligned the normalized firing rates of

604 each neuron at choice execution (when eye-fixation on the chosen offer passed $200 \mathrm{~ms}$ and thus signaled

605 commitment to the choice). Then we took the FR from $1500 \mathrm{~ms}$ before this onset through $1500 \mathrm{~ms}$ after

606 this onset as the choice period (including $1500 \mathrm{~ms}$ pre-choice, outcome delivery, and ITI). We then slid

607 through the offer and the choice periods and generated non-overlapping population activity patterns that

608 were $50 \mathrm{~ms}$ in width and tiled the entire offer and choice periods.

Then we followed a four-fold cross validation procedure, which involved training different LDA

610 decoders on $75 \%$ of the correct trials to differentiate the chosen option (offer 1 vs. 2), the chosen location

611 (left vs. right), and the expected value of offer 1 (EV1 high vs. low) on each trial. Then we tested the

612 decoder on the other $25 \%$ of the correct trials. Decoding accuracy in error trials was obtained by using the

613 same trained LDA decoders to decode all error trials (since none of the error trials were used for training).

614 For EV1 high vs. low, we compared EV1 from each trial to the mean EV of all offers. If the EV1 was

615 larger than or equal to the mean, then it was counted as a high EV1, otherwise low.

618 activities, we first organized our spiking data into population states. We defined the population state as

619 the normalized firing rate of each of all simultaneously recorded neurons, averaged over a $200 \mathrm{~ms}$ time

620 bin, in each region. Then we slid across all time points in each trial with a $50 \mathrm{~ms}$ step size to calculate

621 population states at each sliding step. We calculated these series of population states for two sets of 
622 simultaneously recorded ensembles in OFCin, OFCout, and PCC, one from each subject. We then applied

623 principal component analysis (PCA) to identify a lower-dimensional space to then measure the population

624 dynamics. We first selected and grouped all correct trials based on whether (1) offer 1 or offer 2 was

625 chosen; (2) left or right offer was chosen; and (3) offer 1 was a higher or lower than average value of

626 offers. Then we conducted PCA on the trial averaged population states for each pair of the above-

627 mentioned three pairs of conditions. To make the measures of population dynamics comparable across

628 regions, we defined top-N PC space as the top N principal components that captured at least $70 \%$ of the

629 variance. For subject P, N equals 6 in OFC $\underline{i n}, 5$ in OFCout, and 15 in PCC. For subject S, N equals 3 in

630 OFCin, 5 in OFCout, and 3 in PCC. We then projected trial-averaged or trial-by-trial population states

631 from correct or error trials and each pair of conditions onto this top-N PC space. This projection resulted

632 in pairs of population trajectories corresponding to pairs of conditions in the top-N PC space expanding

633 the whole trial length. We then measured the Euclidean distance at each time point in a trial between the

634 pairs of population trajectories. We used a shuffle procedure in which trials were shuffled across

635 conditions. This shuffle procedure was implemented 1000 times to generate 1000 randomized trial-

636 averaged trajectories for each trial condition, and significance cutoff were set at the top and bottom $2.5 \%$

637 of the shuffled results. For trial-by-trial population state projections that resulted in a pair of two sets of

638 population trajectories (that is, each trajectory corresponded to a specific trial condition), we calculated

639 the adjusted Euclidean distance. The adjusted Euclidean distance is the Euclidean distance across

640 conditions (cross distance) normalized by the Euclidean distance within conditions. Cross distance was

641 defined as the Euclidean distance from one point on one trajectory in one trial condition to all the

642 trajectories' corresponding time point in the other trial condition. Self distance/dispersion was defined as

643 the Euclidean distance of one point on one trajectory in one trial condition to all the other trajectories'

644 corresponding time point in the same trial condition.

645

646 Equation 1: adjusted distance $=\frac{\text { cross distance }}{\text { self distance }}$ 
648 Normalizing the cross distance with self distance controls for the "internal noise level" to make the

649 distance comparable across regions (Murray et al., 2017). The distance, or separation, between population

650 trajectories from pairs of trial conditions represents the population neural activity variance devoted to

651 distinguish those trial conditions (Wang \& Hayden, 2017). Intuitively, it can be interpreted as: the larger

652 the distance/separation between trajectories for different conditions, the more information the variance in

653 this neural population conveys to tell these conditions apart. PCA analysis and Euclidean distance

654 calculation used pca and pdist2 functions in Matlab.

655 

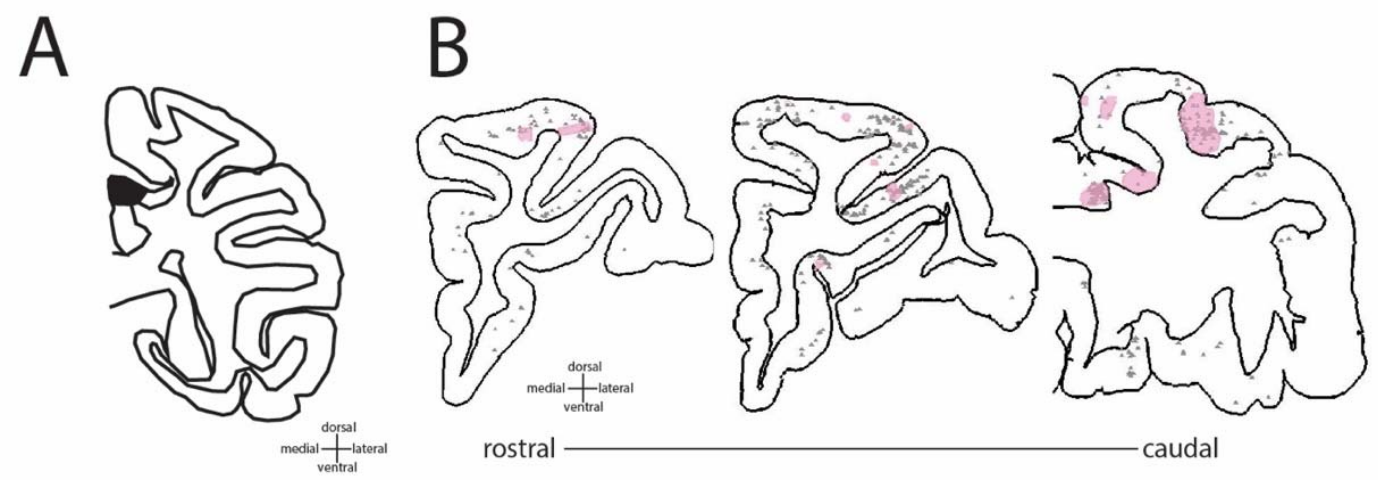

660

Supplementary Figure 1: Anatomical connectivity in prefrontal cortex following an

injection in the PCC sulcus. A. Injection site in PCC B. Terminal field labeling shown in pink; retrograde labeling shown as gray dots.

\section{Behavior of each subject in the gambling task}

We examined the behavior of two male macaque subjects (Macaca mulatta, subjects P and S)

667 performing a well-studied two-option risky choice task (Strait et al., 2014). The data and results we

668 present here have not been published before, but qualitatively replicate our past findings. Specifically,

669 behavioral data indicate that subjects understood the key elements of the task., They preferred offers with

670 the larger expected value on $73.10 \%$ of the trials (for individual subjects, see below). This proportion is

671 significantly higher than expected by chance ( $\mathrm{p}<0.001$, binomial test). It is also quantitatively similar to

672 numbers we have found using the same task in other subjects (Strait et al., 2014; Strait et al., 2015).

673 Subjects' willingness to choose an offer varied as a function of the difference in values between the two

674 offers (Supplementary Figure 2A-B). Both subjects slightly preferred offer 2, although the size of the 
675 effect was small; choosing offer $146.90 \%$ of the time). Note that these behavioral results are restricted to

676 trials in which our physiological recordings met criteria for analysis. Data collected in other sessions were

677 not noticeably different (data not shown).

678 Behaviors of each individual subject closely resembled those of two subjects combined as

679 reported in the main text. Subject P preferred offers with the larger expected value on $73.35 \%$ of the

680 trials. This proportion is significantly higher than expected by chance ( 479 out of $653, \mathrm{p}<0.001$, binomial

681 test). P shifted choices from offer 1 to offer 2 as the expected value difference of offer 1 minus offer 2

682 decreased, even with a slight bias against offer 1 (psychometrics function slightly shifted towards right,

683 choosing offer $145.18 \%$ of the time). Subject S preferred offers with the larger expected value on $72.39 \%$

684 of the trials. This proportion is significantly higher than expected by chance ( 367 out of $507, \mathrm{p}<0.001$,

685 binomial test). S shifted their choice from offer 1 to offer 2 as the expected value difference of offer 1

686 minus offer 2 decreased, even with a slight bias against offer 1 (psychometrics function slightly shifted

687 towards right, choosing offer $145.18 \%$ of the time). 


\section{SF1. Behavior of Each Subject}

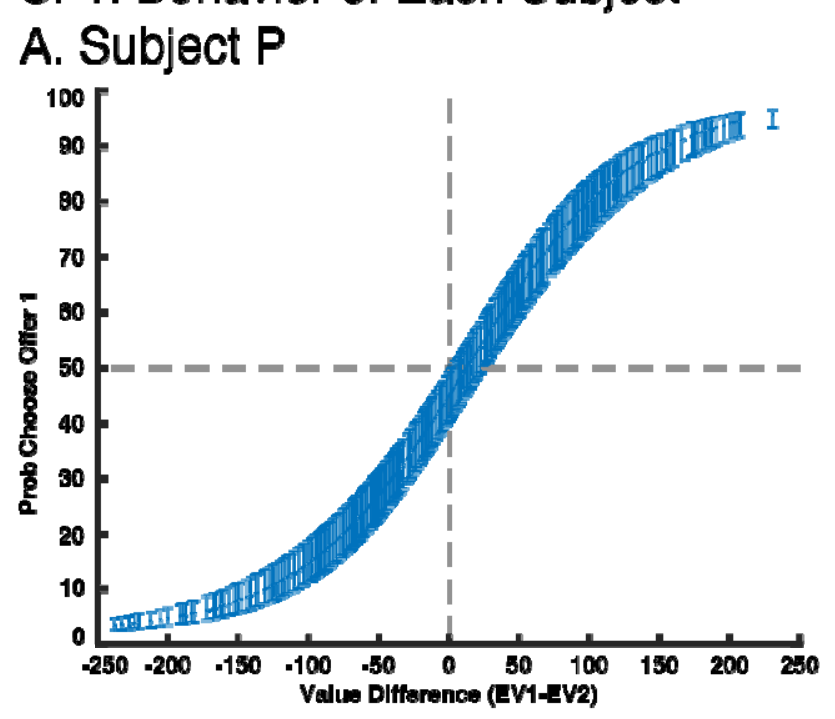

\section{B. Subject S}

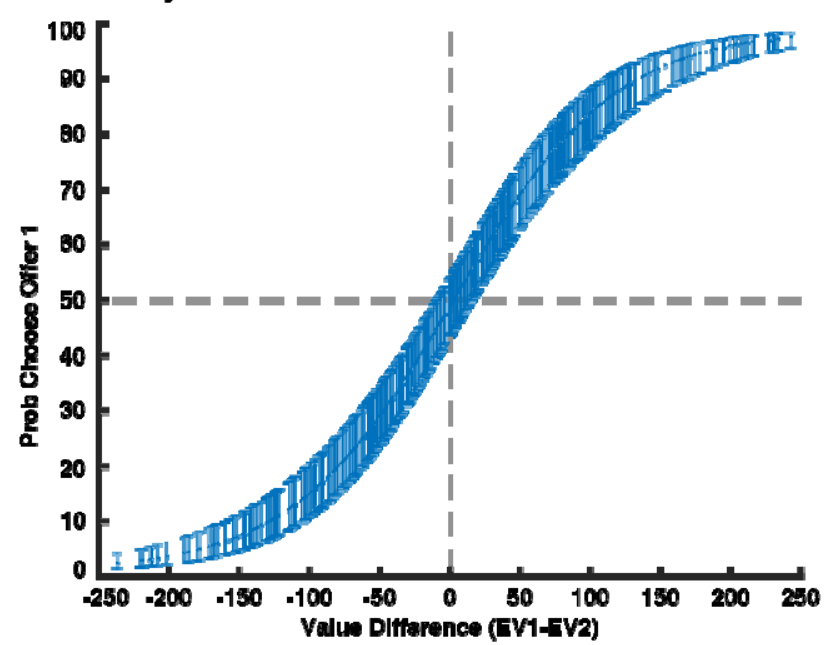

Supplementary Figure 2: Behavior of Each Subject. A. Choices of subject P. B. Choices of subject S. EV, expected value (see Methods). Gray dotted lines represent visual reference for value 0 on $\mathrm{X}$ axis and value 50 on $\mathrm{Y}$ axis. Error bars on the fitted sigmoidal function represents $95 \%$ confidence interval from the model estimation.

We first characterized the local field potentials in each of the OFCin, OFCout, and PCC regions.

697 With multitaper spectral analyses, we show that power peaked around $10 \mathrm{~Hz}$ in OFCin and OFCout, and 
around 10 and $20 \mathrm{~Hz}$ in PCC (Supplementary Figure 3A-C). It also shows that our notch filter effectively removed power around $60 \mathrm{~Hz}$.

The higher coherence in the $\mathrm{OFCin} n_{\mathrm{spk}}-\mathrm{PCC}_{\mathrm{lfp}}$ circuit was also observed within all specific bands that we tested: the delta $(0.5-5 \mathrm{~Hz})$ frequency band $(\mathrm{z}=2.53, \mathrm{p}=0.012)$, the theta $(5-10 \mathrm{~Hz})$ band $(\mathrm{z}=3.55$, $\mathrm{p}<0.001)$, the alpha $(10-15 \mathrm{~Hz})$ band $(\mathrm{z}=3.83, \mathrm{p}<0.001)$, the beta $(15-30 \mathrm{~Hz})$ band $(\mathrm{z}=4.38, \mathrm{p}<0.001)$, and the gamma $(30-100 \mathrm{~Hz})$ band $(\mathrm{z}=5.51 \mathrm{p}<0.001)$. Comparing the coherence for each frequency band within each circuit during offer epoch, there was no significant difference among frequency bands within either the OFCin $n_{\mathrm{spk}}-\mathrm{PCC}_{\mathrm{lfp}}\left(\chi^{2}=3.95, \mathrm{p}=0.413\right.$, Kruskal-Wallis test $)$ or the $\mathrm{OFC}$ out $_{\mathrm{spk}}-\mathrm{PCC}_{\mathrm{lfp}}\left(\chi^{2}=2.28, \mathrm{p}=0.685\right.$, Kruskal-Wallis test) circuit. $(\mathrm{z}=1.41, \mathrm{p}=0.159)$ bands. Comparing the coherence for each frequency band within each circuit, during

711 choice epoch, there was no significant difference among frequency bands within either OFCin $n_{\mathrm{spk}}-\mathrm{PCC}_{\mathrm{lfp}}$ $712\left(\chi^{2}=1.81, \mathrm{p}=0.771\right.$, Kruskal-Wallis test $)$ or $\mathrm{OFC}_{\text {out }} \mathrm{spk}-\mathrm{PCC}_{\mathrm{lfp}}\left(\chi^{2}=2.53, \mathrm{p}=0.640\right.$, Kruskal-Wallis test $)$.

714 coherence OFCin $_{\text {spk }}-\mathrm{PCC}_{\text {lfp }}$ than in $\mathrm{OFC}$ out $_{\mathrm{spk}}-\mathrm{PCC}_{\text {lfp }}$ circuit, within the delta $(\mathrm{z}=3.36, \mathrm{p}<0.001)$, the

715 theta $(\mathrm{z}=2.87, \mathrm{p}=0.004)$, the alpha $(\mathrm{z}=3.70, \mathrm{p}=0.002)$, and the gamma $(\mathrm{z}=2.05, \mathrm{p}=0.040)$ bands, although

716 not within the beta $(\mathrm{z}=1.27, \mathrm{p}=0.204)$ band. Comparing the overall coherence for each frequency band

717 during reward epoch, there was significant difference among frequency bands within OFCin $n_{\mathrm{spk}}-\mathrm{PCC}_{\mathrm{lfp}}$

$718\left(\chi^{2}=14.32, \mathrm{p}=0.006\right.$, Kruskal-Wallis test with Tukey-Kramer multiple comparison $)$ circuit. Specifically,

719 within $\mathrm{OFCin}_{\mathrm{spk}}-\mathrm{PCC}_{\mathrm{lfp}}$, the coherence in the beta band was significantly lower than that in the theta

720 band $(\mathrm{p}=0.021)$ and that in the alpha band $(\mathrm{p}=0.009)$. Similarly, comparing the overall coherence for each

721 frequency band during the reward epoch, there was a significant difference among frequency bands

722 within OFCout spk $_{\text {PCC }}$ Pfp circuit $\left(\chi^{2}=17.15, \mathrm{p}=0.002\right.$, Kruskal-Wallis test with Tukey-Kramer multiple 
723 comparison). Specifically, within $\mathrm{OFCout}_{\mathrm{spk}}-\mathrm{PCC}_{\mathrm{lfp}}$, the coherence in the alpha band was significantly

724 lower than that in the theta band $(\mathrm{p}=0.007)$ and that in the gamma band $(\mathrm{p}=0.029)$.

725 Together, we found greater coherence in OFCin spk $_{-}-\mathrm{PCC}_{\mathrm{lfp}}$ than in OFCout $t_{\mathrm{spk}}-\mathrm{PCC}_{\mathrm{lfp}}$, suggesting

726 stronger functional connectivity. This pattern of enhanced coherence was not found in the reverse

727 direction (that is, in the $\mathrm{PCC}_{\mathrm{spk}}-\mathrm{OFCin}_{\mathrm{lfp}}, \mathrm{PCC}_{\mathrm{spk}}-\mathrm{OFC}$ out $t_{\mathrm{lf}}$ circuits) or their comparison

728 (Supplementary Figure 3D-J).

729 We further compared the broadband spike-field coherence in the reverse direction to that reported

730 in the main text. We found significantly higher broadband coherence in $\mathrm{OFCin}_{\mathrm{spk}}-\mathrm{PCC}_{\mathrm{lfp}}$ than $\mathrm{PCC}_{\mathrm{spk}}$ -

$731 \mathrm{OFCin}_{\mathrm{lfp}}(\mathrm{z}=4.83, \mathrm{p}<0.001$, Wilcoxon signed rank test, Supplementary Figure 3D). The broadband

732 coherence was also higher in $\mathrm{OFCout}_{\mathrm{spk}}-\mathrm{PCC}_{\mathrm{lfp}}$ than in $\mathrm{PCC}_{\mathrm{spk}}-\mathrm{OFCout}_{\mathrm{lfp}}(\mathrm{z}=2.90, \mathrm{p}=0.004$, Wilcoxon

733 signed rank test, Supplementary Figure 3E). We also found significantly higher broadband coherence in

$734 \mathrm{PCC}_{\mathrm{spk}}-\mathrm{OFC}_{\mathrm{lfp}}$ than in $\mathrm{PCC}_{\mathrm{spk}}-\mathrm{OFCout}_{\mathrm{lfp}}(\mathrm{z}=2.76, \mathrm{p}=0.006$, Wilcoxon signed rank test;

735 Supplementary Figure 3D-E) but no significant differences in broadband coherence between OFCin spk $^{-}$

736 OFCout $_{\mathrm{lfp}}$ and OFCout spk $_{-}-$OFCin $_{\mathrm{lfp}}(\mathrm{z}=0.15, \mathrm{p}=0.883$, Wilcoxon signed rank test; Supplementary

737 Figure 3F-G).

738 Spike-field coherence is theorized to capture long-range input from the spiking region to the field

739 region. Our results suggest that the enhanced synchronization for OFCin-PCC could be dominated by

740 OFCin's input to influencing PCC local neurocomputation. 
SF2. Funotional Conneotlvity.
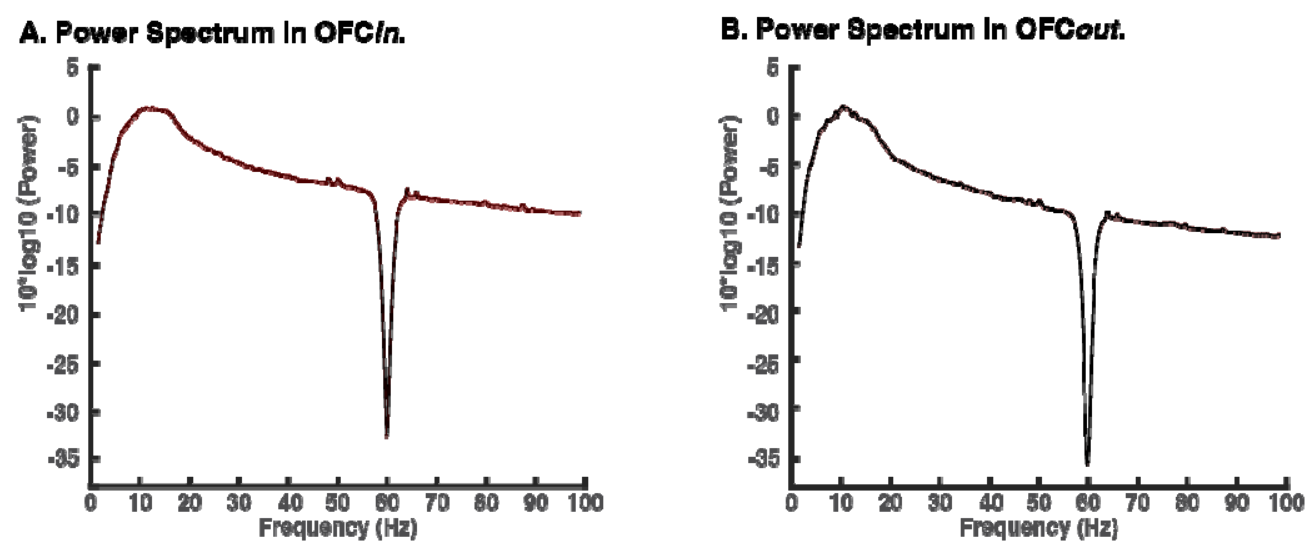

C. Power Speotrum In PCC.

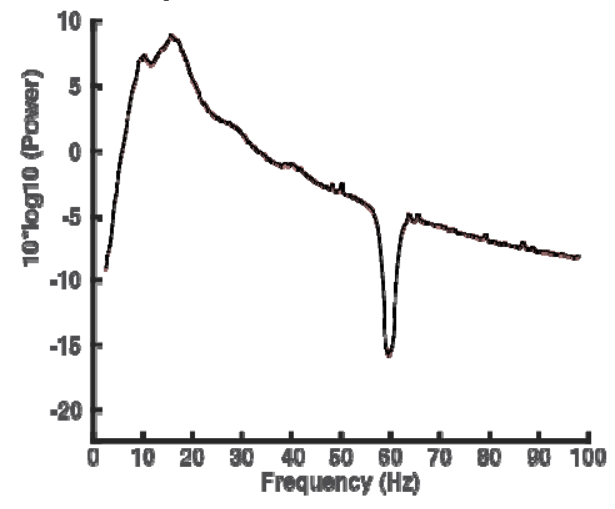

D. Splke (PCC) - Fleld (OFC/n) Coherenee.

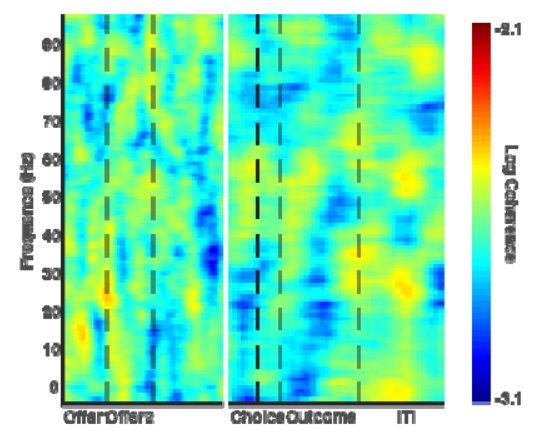

E. Splke (PCC) - Fleld (OFCout) Cohørene⿻.

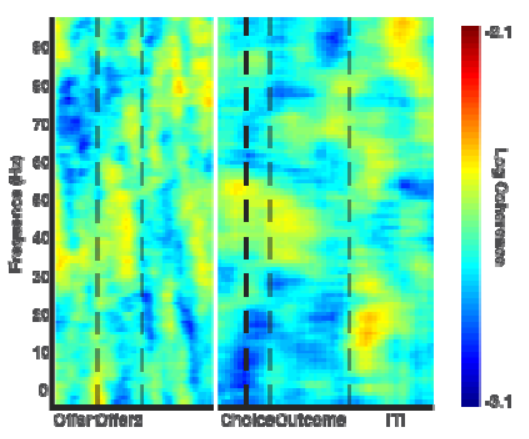

\section{F. 8plke (OFCln) - Fleld (OFCout) Coherence.}

\section{G. 8plke (OFCout) - Fleld (OFCIn) Coherence.}
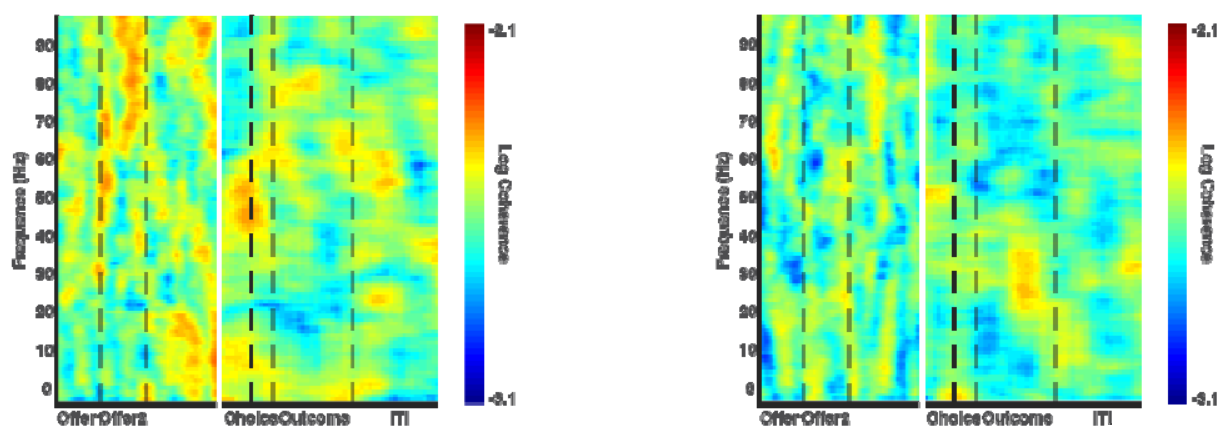

spectrum in OFCin (A), in OFCout (B), and in PCC (C). X axis: frequency $(\mathrm{Hz})$. Y axis: power transformed with a $10 \times \log 10$ function. Black line: mean power across channel and across trials. Red shaded area: 95\% confidence interval. D-J. Spike-field coherence. $X$ axis: time in a trial. Y axis: frequency. Color: strength of spike-field coherence on log10 scale. The warmer the color, the higher the coherence. Data from the first half of the trial (offer period) was aligned at offer 1 onset. Data from the second half of the trial (choice period) was aligned at Choice execution. (D) $\mathrm{PCC}_{\mathrm{spk}}-\mathrm{OFC} i n_{\mathrm{lfp}}$ coherence. (E) PCC $\mathrm{spk}^{-} \mathrm{OFCout} \mathrm{t}_{\mathrm{ff}}$ coherence. (F) OFCin $\mathrm{spk}^{-}$ OFCout $_{\text {lfp }}$ coherence. (G) OFCout $t_{\text {spk }}-$ OFCin ${ }_{\text {lfp }}$ coherence.

\section{Greater mutual information between OFCin-PCC and OFCout-PCC circuits}

$755 \quad\left(\mathrm{z}=17.47, \mathrm{p}<0.001\right.$, Wilcoxon signed rank test). Specifically, the OFCin-PCC circuit shared $7.44 \times 10^{-4}$ bits

756 of information per channel, while the OFCout-PCC circuit shared $6.72 \times 10^{-4}$ bits per channel. This

757 difference was observed during the offer 1 epoch $(\mathrm{z}=8.81, \mathrm{p}<0.001)$, during the offer 2 epoch $(\mathrm{z}=8.34$,

$758 \mathrm{p}<0.001)$, during the choice epoch $(\mathrm{z}=9.42, \mathrm{p}<0.001)$, and during the reward epoch $(\mathrm{z}=8.23, \mathrm{p}<0.001)$.

759 The difference was not observed during the inter-trial interval epoch (ITI, $z=0.71, p=0.479$ ).

(Methods). All three regions encoded offer and outcome values with similar proportion of neurons,

765 encoding strength, and latencies. neurons, $16.67 \%(\mathrm{n}=9 / 54, \mathrm{p}=0.001)$ of OFCout neurons, and $13.62 \%(\mathrm{n}=29 / 213, \mathrm{p}<0.001)$ of PCC

768 neurons encoded the value of offer 1 . These proportions were not detectably different from one another $769\left(\chi^{2}=0.79, \mathrm{df}=2, \mathrm{p}=0.675\right.$, Chi-square test $)$. 
772 OFCin, OFCout, and PCC $\left(\chi^{2}=1.67, \mathrm{p}=0.434\right.$, Kruskal-Wallis test $)$. We then assessed response latencies

773 using a generalized linear model with Gamma distribution (Bishop, 2006; MacKay, 2003). For the

774 latency analysis, we used all neurons, because many neurons in a population can show encoding of task

775 variables without passing statistical significance; considering all neurons improves accuracy. Among all

776 neurons, the encoding strength of offer 1 value peaked at $290 \mathrm{~ms}$ in OFCin, $235 \mathrm{~ms}$ in OFCout, and 240

$777 \mathrm{~ms}$ in PCC, after offer 1 onset. We then used the distributions of single-neuron latencies to assess

778 statistical significance; by this method, these latencies were not significantly different from one another

$779 \quad(\mathrm{~F}=1.39, \mathrm{p}=0.251$, GLM with Gamma distribution; Methods).

780 During the outcome epoch, $34.09 \%$ ( $\mathrm{n}=15 / 44, \mathrm{p}<0.001$, binomial test) of OFCin neurons, $35.19 \%$

$781(\mathrm{n}=19 / 54, \mathrm{p}<0.001$, binomial test $)$ of OFCout neurons, and 52.58\% (n=112/213, $\mathrm{p}<0.001$, binomial test $)$

782 of PCC neurons encoded the value of received outcome. The proportion of such neurons in PCC was

783 significantly higher than those of OFCin and OFCout $\left(\chi^{2}=8.63, \mathrm{df}=2, \mathrm{p}=0.013\right.$, Chi-square test, cf.

784 Hayden et al., 2008). The encoding strength of outcome value at the population level was significantly

785 higher in PCC than both OFCin and OFCout $\left(\chi^{2}=9.83, \mathrm{p}=0.007\right.$, Kruskal-Wallis test with Tukey-Kramer

786 multiple comparison). The encoding of outcome value peaked around $275 \mathrm{~ms}$ in OFCin, $360 \mathrm{~ms}$ in

787 OFCout, and $450 \mathrm{~ms}$ in PCC, after reward onset. These latencies were not significantly different from one 788 another $(\mathrm{F}=1.30, \mathrm{p}=0.275$, GLM with Gamma distribution).

789 All three regions encoded chosen option (offer 1 vs. 2) and chosen location (left vs. right).

790 However, OFCin encoded the chosen option with shorter latency than both OFCout and PCC. PCC, not

791 OFCin nor OFCout, showed a higher proportion of neurons encoding chosen location than chosen option.

792 PCC and OFCin also encoded the chosen location with significantly shorter latencies than OFCout.

793 We defined choice epoch as the period from $200 \mathrm{~ms}$ after offer 2 was presented until when choice

794 was made via saccade and fixation on the chosen option. During this time, $18.18 \%(\mathrm{n}=8 / 44, \mathrm{p}=0.001$,

795 binomial test) of OFCin neurons, $16.67 \%$ ( $\mathrm{n}=9 / 54, \mathrm{p}=0.001$, binomial test) of OFCout neurons, and

$79612.21 \%(n=26 / 213, p=0.001$, binomial test $)$ of PCC neurons encoded chosen option (offer 1 vs. 2). These 
797 proportions were not significantly different from one another $\left(\chi^{2}=2.62, \mathrm{df}=2, \mathrm{p}=0.270\right.$, Chi-square test $)$.

798 Encoding strength of chosen option at population level was not significantly different across regions

$799\left(\chi^{2}=1.35, \mathrm{p}=0.510\right.$, Kruskal-Wallis test $)$. The encoding of chosen option peaked at $90 \mathrm{~ms}$ in OFCin, 170

$800 \mathrm{~ms}$ in OFCout, and $150 \mathrm{~ms}$ in PCC into choice epoch. These latencies were significantly different from

801 one another $(\mathrm{F}=3.35, \mathrm{p}=0.037$, GLM with Gamma distribution). Specifically, OFCin latency was

802 significantly shorter than that in OFCout $(\mathrm{t}=-2.14, \mathrm{p}=0.033$, from the same GLM fit $)$ or PCC $(\mathrm{t}=-2.36$,

$803 \mathrm{p}=0.019$, from the same GLM fit), but there was no significant difference between OFCout and PCC

$804(\mathrm{t}=0.12, \mathrm{p}=0.906$, from the same GLM fit).

805 During the same choice epoch, $18.18 \%(\mathrm{n}=8 / 44, \mathrm{p}=0.001$, binomial test $)$ of OFCin neurons,

$80612.96 \%(n=7 / 54, p=0.018$, binomial test $)$ of OFCout neurons, and 19.25\% $(n=41 / 213, p<0.001$, binomial

807 test) of PCC neurons encoded chosen location (left vs. right). These proportions were not significantly

808 different from one another $\left(\chi^{2}=1.15, \mathrm{df}=2, \mathrm{p}=0.562\right.$, Chi-square test $)$. However, $\operatorname{PCC}\left(\chi^{2}=5.31, \mathrm{df}=1\right.$,

$809 \mathrm{p}=0.021$, Chi-square test $)$ but not OFCin $\left(\chi^{2}=0, \mathrm{df}=1, \mathrm{p}=1\right.$, Chi-square test $)$ or OFCout $\left(\chi^{2}=0.07, \mathrm{df}=1\right.$,

$810 \mathrm{p}=0.787$, Chi-square test) showed a higher proportion of neurons encoding chosen location than chosen

811 option. Encoding strength of chosen location at the population level was not significantly different across

812 the three regions $\left(\chi^{2}=0.20, p=0.906\right.$, Kruskal-Wallis test $)$. The encoding of chosen location peaked around

$813150 \mathrm{~ms}$ in OFCin, $230 \mathrm{~ms}$ in OFCout, and $140 \mathrm{~ms}$ in PCC, into the choice epoch. These latencies were

814 significantly different from one another ( $\mathrm{F}=5.71, \mathrm{p}=0.004$, GLM with Gamma distribution). Specifically,

815 OFCout latency was significantly longer than that in OFCin ( $\mathrm{t}=2.36, \mathrm{p}=0.019$, from the same GLM fit)

816 and $\mathrm{PCC}(\mathrm{t}=3.47, \mathrm{p}<0.001$, from the same GLM fit), but there was no significant difference between those

817 in OFCin and PCC ( $\mathrm{t}=0.07, \mathrm{p}=0.944$, from the same GLM fit $)$.

\section{Functional differences in decoding between OFCin-PCC and OFCout-PCC circuits}

820 We then asked whether the relay of choice signal from value space to action space can be

821 observed in decodability from population activities across all three regions. To answer this question, we 
822 took the normalized firing rate of each neuron over a sliding window to get the population activity pattern

823 from all simultaneously recorded neurons in each trial. Then we trained a linear discriminant analysis

824 (LDA) decoder on the population activity patterns from $75 \%$ of the trials and tested the decoder on the

825 remaining $25 \%$ of the trials following a four-fold cross-validation procedure (Methods).

826 We found that at the end of offer 2 presentation (500 $\mathrm{ms}$ epoch), the value of the chosen option

827 (offer 1 vs. 2 ) was decodable in all three of OFCin $\left(\chi^{2}=7.41, \mathrm{p}=0.006\right.$, chi-square test), OFCout $\left(\chi^{2}=5.63\right.$,

$828 \mathrm{p}=0.018)$, and PCC $\left(\chi^{2}=12.45, \mathrm{p}<0.001\right)$, on correct trials. These three proportions were not significantly

829 different from one another $\left(\chi^{2}=1.41, \mathrm{p}=0.494\right)$, suggesting the decodability was similar across regions.

830 The value of the chosen options was not decodable on error trials in OFCin $\left(\chi^{2}=0.57, \mathrm{p}=0.448\right)$ or PCC

$831\left(\chi^{2}=0.25, p=0.613\right)$, although it was decodable in OFCout $\left(\chi^{2}=6.83, p=0.009\right)$ (Supplementary Figure

832 5A). Right before a saccade was used to select the chosen option, chosen location (left vs. right) was not

833 decodable in OFCout $\left(\chi^{2}=0.02, \mathrm{p}=0.901\right)$, but was decodable in OFCin $\left(\chi^{2}=0.25, \mathrm{p}=0.049\right)$ and PCC

$834\left(\chi^{2}=8.85, \mathrm{p}=0.003,\right)$, on correct trials. These three proportions were significantly different from one

835 another $\left(\chi^{2}=8.37, \mathrm{p}=0.015\right)$; the proportion in PCC was significantly higher than in OFCin $\left(\chi^{2}=8.12\right.$,

$836 \mathrm{p}=0.004)$. Chosen location was not decodable on error trials in OFCin $\left(\chi^{2}=0.30, \mathrm{p}=0.584\right.$, OFCout $\left(\chi^{2}=0\right.$,

$837 \mathrm{p}=1)$, or PCC $\left(\chi^{2}=0.06, \mathrm{p}=0.801\right.$; Supplementary Figure 5B $)$.

838 As a control test, we also tested decoding accuracy of EV1 high vs. low value. As shown in

839 (Supplementary Figure 5C-D), both circuits / all three regions showed slightly but significantly higher

840 than chance levels of decoding accuracy for whether EV1 was high or low in correct trials. Interestingly,

841 decoding accuracies were not significantly different from the chance level in error trials during the offer

842 period, and only reached slightly higher than chance level during outcome delivery.

843 Decodability for chosen location (left vs. right) was particularly prominent in PCC and was

844 quenched on error trials. In addition, the decodability for chosen option (offer 1 vs. 2) in OFCin was also

845 quenched on error trials. We wondered whether information in OFCin for chosen option, after being read

846 out (decoded) by PCC, would influence the decodability of chosen location in PCC. In other words, does 
847 the relay of choice in value space to that in action space happen through the information input from

848 OFCin to PCC, and can it be read out from PCC? To answer this question, we applied the Granger

849 causality test to the decodability of chosen option and chosen location across regions.

850 We found that the decodability for chosen option (offer 1 vs. offer 2) in OFCin Granger-caused

851 the decodability for chosen location (left vs. right) in PCC ( $\mathrm{gc}=11.19, \mathrm{p}=0.025)$ with a $200 \mathrm{~ms}(5 \mathrm{~Hz})$ lag.

852 This Granger-causal relation was absent on error trials $(\mathrm{gc}=3.04, \mathrm{p}=0.552)$, suggesting the successful

853 transform of choice signal in these two frameworks might be crucial for correct choice behavior. In the

854 reverse direction, the decodability for chosen location (left vs. right) in PCC Granger-caused decodability

855 for chosen option (offer 1 vs. 2) in OFCin $(g c=17.59, \mathrm{p}=0.025)$ but with a much longer time lag (400 ms;

$8562.5 \mathrm{~Hz}$ ). In contrast, the decodability for chosen option (offer 1 vs. 2) in OFCout did not Granger-cause

857 the decodability for chosen location (left vs. right) in PCC at any time lag, nor did the chosen location

858 (left vs. right) in OFCin at any time lag.

Functional differences in population dynamics between OFCin-PCC and OFCout-PCC circuits

After projecting trial-by-trial population states onto the top-N PC space, we found that in error

trials, the overall distance between trial-by-trial population trajectories for chosen option (offer 1vs2) was

863 significantly different across OFCin, OFCout, and PCC $\left(\chi^{2}=59.88, \mathrm{p}<0.001\right.$, Kruskal-Wallis test with

864 Tukey-Kramer multiple comparison), with distance in OFCout significantly higher than in OFCin

$865(\mathrm{p}=0.036)$ and PCC $(\mathrm{p}<0.001)$ and distance in OFCin significantly higher than in PCC $(\mathrm{p}<0.001)$. The

866 overall distance in error trials between trial-by-trial population trajectories for chosen location (left vs

867 right) was not significantly different across OFCin, OFCout, and PCC $\left(\chi^{2}=3.95, p=0.139\right)$. The overall

868 distance between trial-by-trial population trajectories for high vs. low EV1 were significantly different

869 across OFCin, OFCout, and PCC $\left(\chi^{2}=8.83, \mathrm{p}=0.012\right)$, with distance in OFCin significantly higher than

870 that in OFCout $(\mathrm{p}=0.033)$ and PCC $(\mathrm{p}=0.023)$ but with no significant difference between OFCout and

871 PCC $(\mathrm{p}=0.990)$. 
873 and error trials for different pairs of task parameters. In OFCin, adjusted distances between population

874 trajectories for chosen option (offer $1 \mathrm{vs} 2 ; \chi^{2}=61.82, \mathrm{p}<0.001$ ), chosen location (left vs right; $\chi^{2}=111.99$,

$875 \mathrm{p}<0.001$ ), and EV1 (high vs low; $\chi^{2}=120.63$, $\mathrm{p}<0.001$ ) were significantly larger in correct than in error

876 trials. In OFCout adjusted distances between population trajectories for chosen option (offer 1vs2;

$877 \quad \chi^{2}=29.37, \mathrm{p}<0.001$ ), chosen location (left vs right; $\chi^{2}=117.80, \mathrm{p}<0.001$ ), and EV1 (high vs low;

$\left.878 \chi^{2}=137.78, \mathrm{p}<0.001\right)$ were significantly larger in correct than in error trials. Similarly, in PCC, adjusted

879 distances between population trajectories for chosen option (offer 1 vs offer $2 ; \chi^{2}=93.01, p<0.001$ ), chosen

880 location (left vs right; $\chi^{2}=137.49, \mathrm{p}<0.001$ ), and EV1 (high vs low; $\chi^{2}=149.19, \mathrm{p}<0.001$ ) were

881 significantly larger in correct than in error trials.

882 Simultaneously, all three regions showed larger dispersion (within-condition distance; see

883 Methods) in error than in correct trials (Supplementary Figure 6). In OFCin, dispersion between

884 population trajectories for chosen option (offer $1 \mathrm{vs} 2 ; \chi^{2}=149.13, \mathrm{p}<0.001$, Kruskal-Wallis test), chosen

885 location (left vs right; $\chi^{2}=149.25, \mathrm{p}<0.001$ ), and EV1 (high vs low; $\chi^{2}=149.25, \mathrm{p}<0.001$ ) were

886 significantly larger in error than in correct trials. In OFCout, dispersion between population trajectories

887 for chosen option (offer $1 \mathrm{vs} 2 ; \chi^{2}=149.25, \mathrm{p}<0.001$ ), chosen location (left vs right; $\chi^{2}=149.25, \mathrm{p}<0.001$ ),

888 and EV1 (high vs low; $\chi^{2}=149.25, \mathrm{p}<0.001$ ) were significantly larger in error than in correct trials.

889 Similarly, in PCC, dispersion between population trajectories for chosen option (offer $1 \mathrm{vs} 2 ; \chi^{2}=149.25$,

$890 \mathrm{p}<0.001$ ), chosen location (left vs right; $\chi^{2}=149.25, \mathrm{p}<0.001$ ), and EV1 (high vs low; $\chi^{2}=149.25$,

$891 \mathrm{p}<0.001)$ were significantly larger in error than in correct trials. 

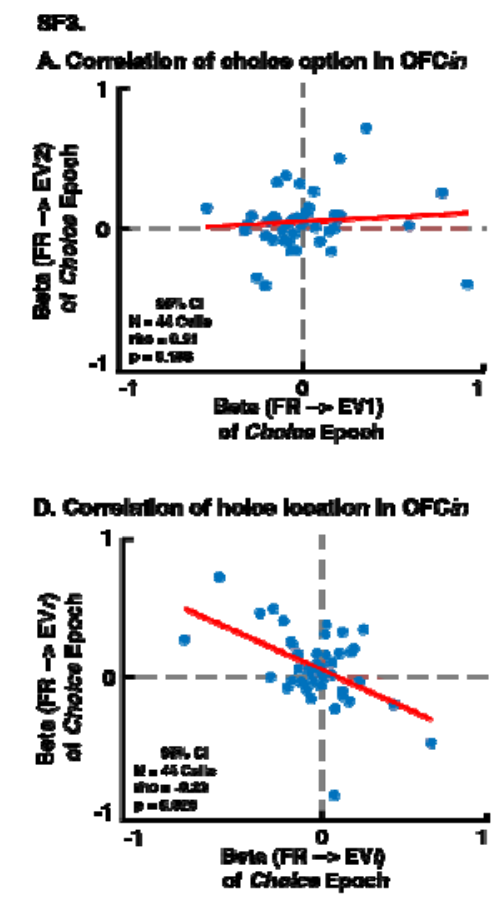
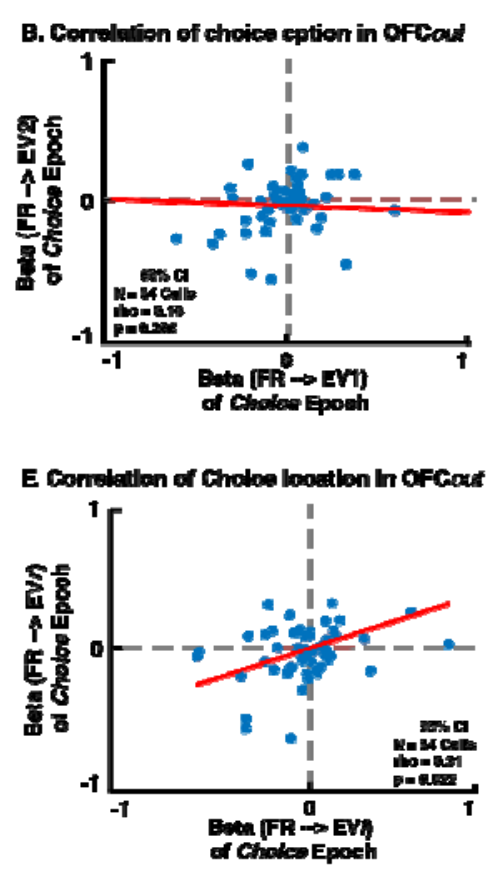

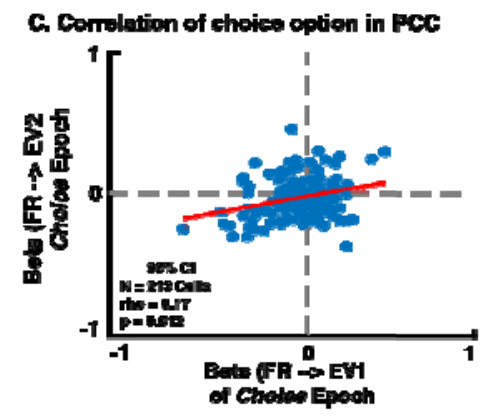

F. Corrolation of Cholee loemilon In PCC.

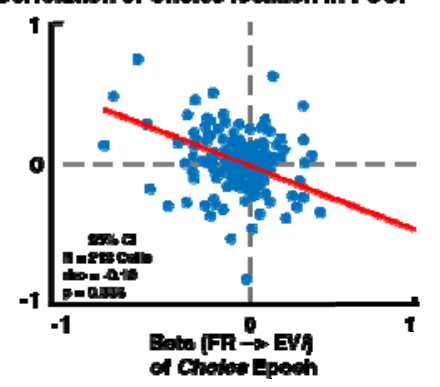

893 Supplementary Figure 4. Putative mutual inhibition effects. A-F: Scatter plots. Each dot represents one neuron. Shaded area: 95\% confidence interval. A-C: Y-axis: regression coefficient for expected value of offer 2. X-axis: regression coefficient for expected value of offer 1. D-F: Y-axis: regression coefficient for expected value of right offer. $\mathrm{X}$-axis: regression coefficient for expected value of left offer. A,D: OFCin. B,E: OFCout. C,F: PCC. These figures are complementary to Figure $3 \mathrm{~A}-\mathrm{F}$ (main text) in that they are results from the same analysis in a later time window (from choice epoch instead of offer 2 epoch), to show the change and development of mutual inhibition signal. 


\section{A. Decoding Accuracy of Choice Option (Error Trials)}

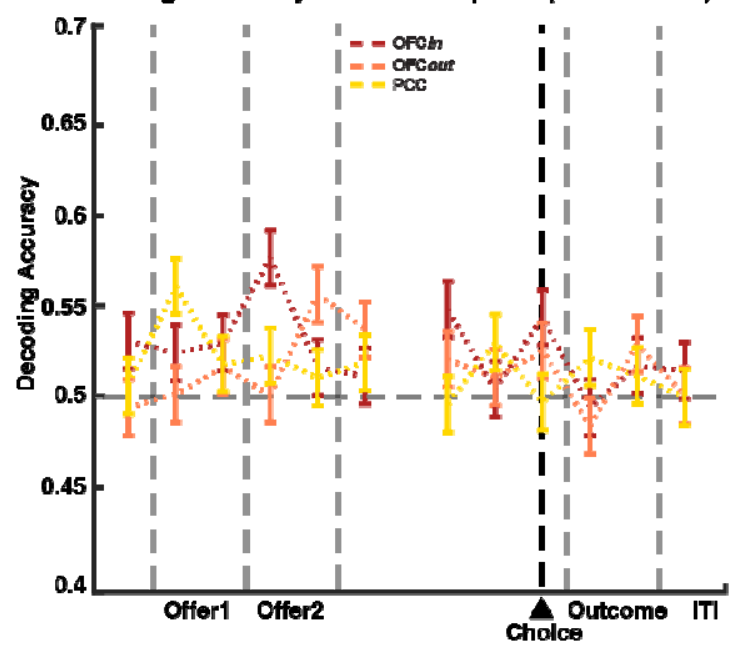

\section{Decoding Accuracy of EV1 High vs. Low (Correct Trials)}

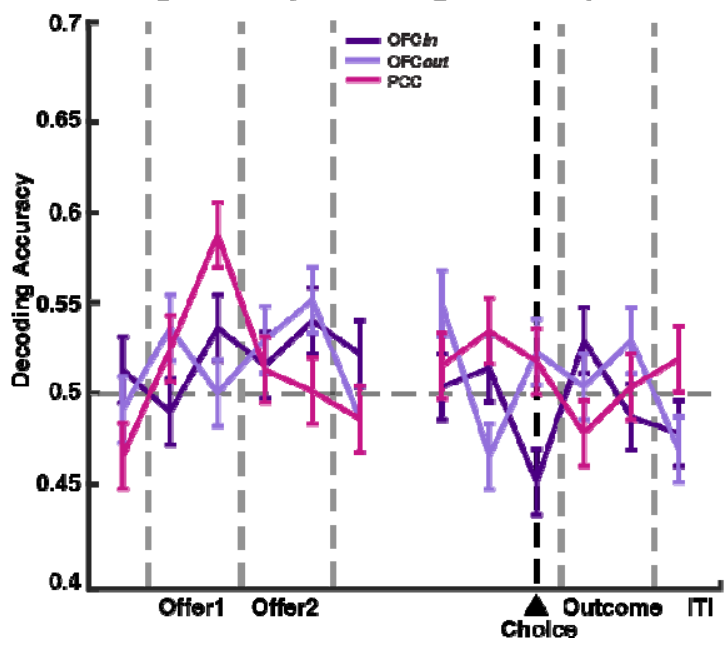

\section{B. Decoding Accuracy of Choice Location (Error Trials)}

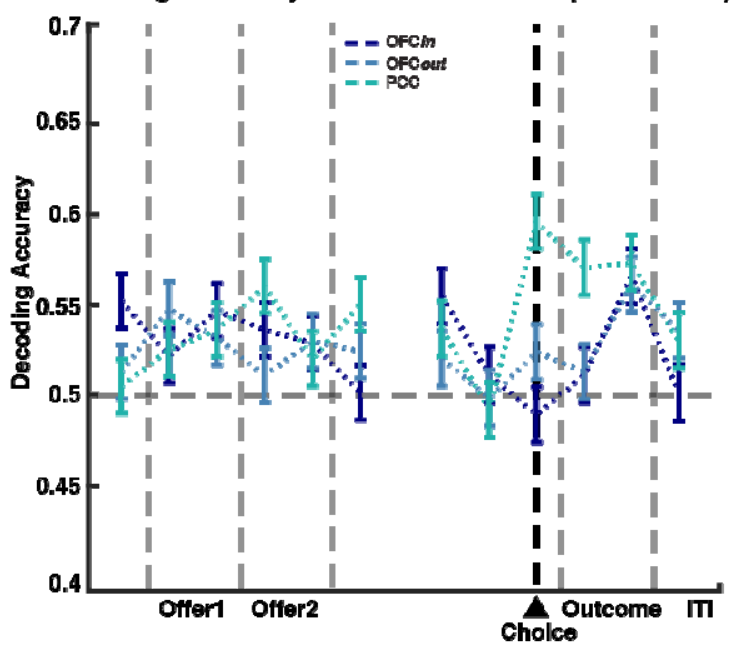

D. Decoding Accuracy of EV1 High v8. Low (Error Trials)

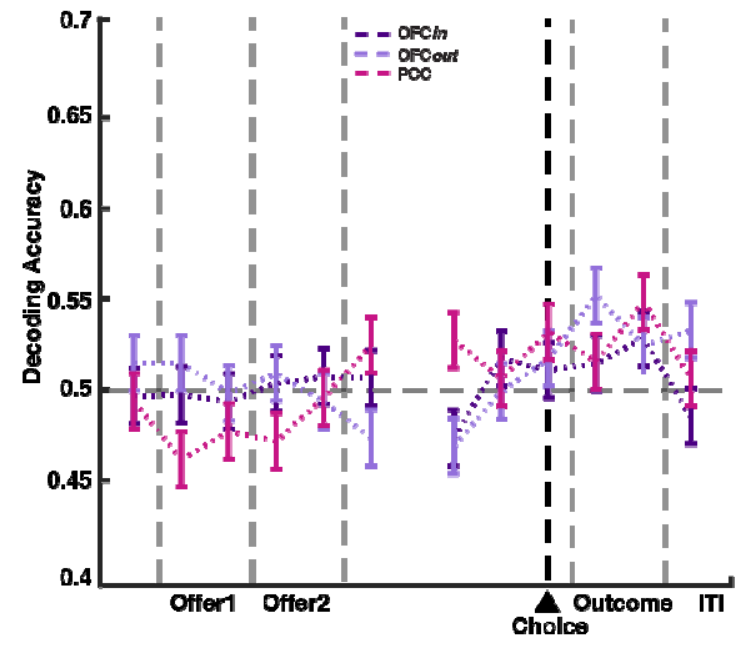

Supplementary Figure 5: Decoding accuracy. A-D: Y-axis: probability of decoding correctly. $X$-axis: time in a trial. Error bar: standard error of the mean. A: Decoding accuracy of choice option (offer 1 vs. offer 2) from error trial (choosing the offer with the smaller expected value). B: Decoding accuracy of choice location (left vs. right) from error trials. C-D: Decoding accuracy of whether the expected value of offer 1 was higher or lower than the average expected value of offer 1 from correct (C) and error (D) trials, respectively. 
911

912

913

914

915

916

917

918

919

920

921

922
B
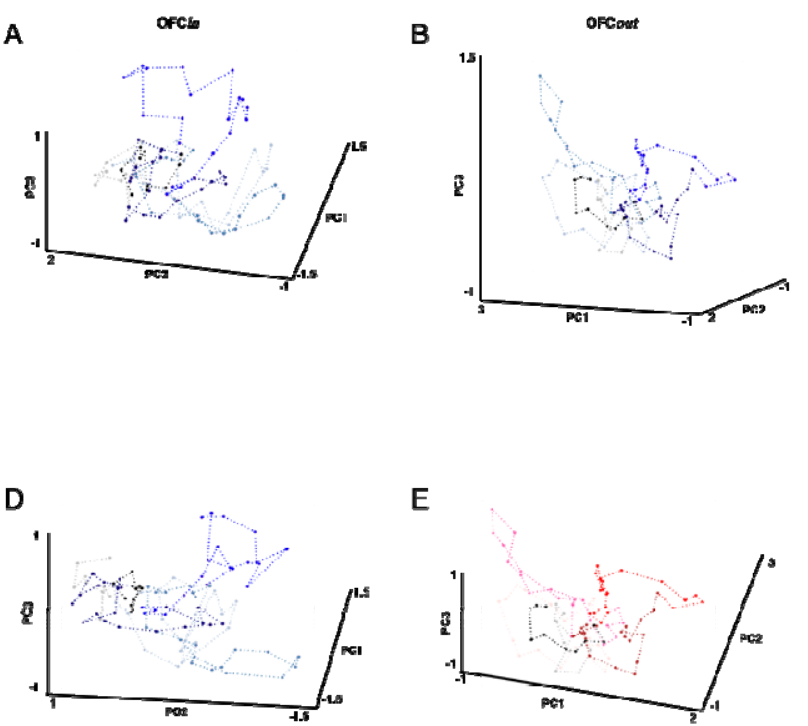

G

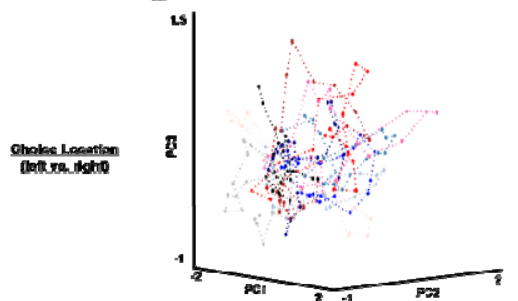

A

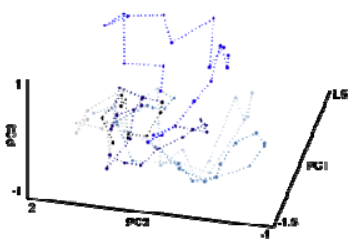

E

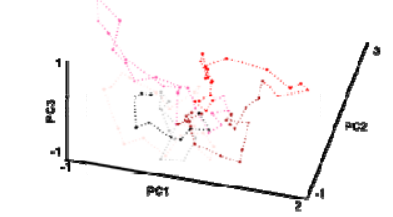

H

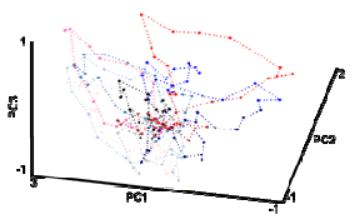

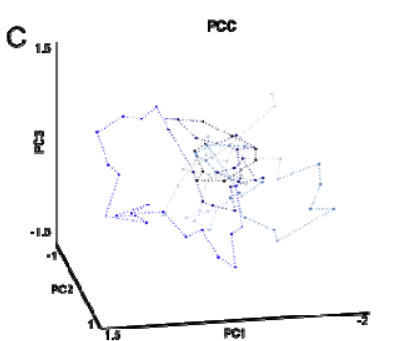

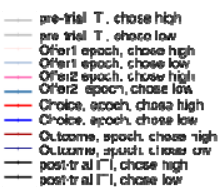

F
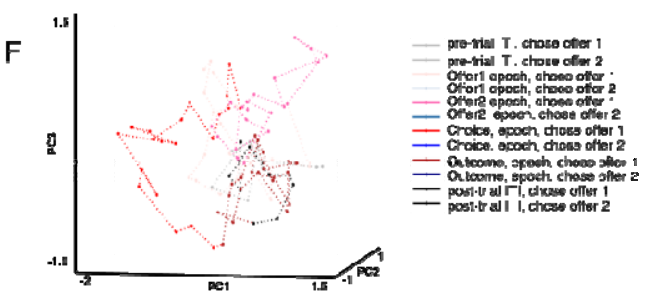

I

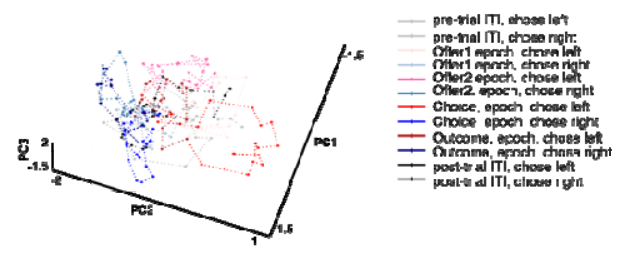

Supplementary Figure 6: Population dynamics on error trials. Error trials are those in which the subject chose the offer with the smaller expected value. Trial averaged population activity projected onto top-N PC space (only top-3 PCs are shown here), separated by EV1 high vs. low (a-c), chosen option (offer 1 vs. offer 2; d-f), and chosen location (left vs. right; g-i), in OFCin (left column), OFCout (middle column), and PCC (right column). Warm color: trial averaged population activity for high EV1 (A-C), choosing offer 1 (D-F) or left offer (G-I). Cold color: trial averaged population activity for low EV1 (A-C), choosing offer 2 (D-F) or right offer (G-I). These figures are complementary to Figure 4. They show that the separate of trajectories based on EV1 and choices are diminished on error trials. 
925 Afshar, A., Santhanam, G., Yu, B. M., Ryu, S. I., Sahani, M., \& Shenoy, K. V. (2011). Single-Trial

$926 \quad$ Neural Correlates of Arm Movement Preparation. Neuron, 71(3), 555-564.

927 Azab, H., \& Hayden, B. Y. (2017). Correlates of decisional dynamics in the dorsal anterior cingulate $928 \quad$ cortex. PLoS biology, 15(11), e2003091.

929 Azab, H., \& Hayden, B. Y. (2018). Correlates of economic decisions in the dorsal and subgenual anterior $930 \quad$ cingulate cortices. European Journal of Neuroscience, 47(8), 979-993.

931 Barack, D. L., Chang, S. W. C., \& Platt, M. L. (2017). Posterior Cingulate Neurons Dynamically Signal 932 Decisions to Disengage during Foraging. Neuron, 96(2), 339-347.e5.

933 Bartolo, R., \& Averbeck, B. B. (2020). Prefrontal Cortex Predicts State Switches during Reversal $934 \quad$ Learning. Neuron, 1-11.

935 Bishop, C. M. (2006). Pattern Recoginiton and Machine Learning. In Information Science and Statistics.

936 Blanchard, T. C., \& Hayden, B. Y. (2014). Neurons in dorsal anterior cingulate cortex signal

937 postdecisional variables in a foraging task. Journal of Neuroscience, 34(2), 646-655.

938 Bokil, H., Andrews, P., Kulkarni, J. E., Mehta, S., \& Mitra, P. P. (2010). Chronux: A platform for

939 analyzing neural signals. Journal of Neuroscience Methods, 192(1), 146-151.

940 Bradfield, L. A., \& Hart, G. (2020). Rodent medial and lateral orbitofrontal cortices represent unique 941 components of cognitive maps of task space. Neuroscience and Biobehavioral Reviews, 108(June 942 2019), 287-294.

943 Brainard, D. H. (1997). The psychophysics toolbox. Spatial vision, 10(4), 433-436.

944 Buzsáki, G. (2004). Large-scale recording of neuronal ensembles. Nature Neuroscience, 7(5), 446-451.

945 Buzsaki, G., \& Draguhn, A. (2004). Neuronal oscillations in cortical networks. Science, 304(5679),

$946 \quad 1926-1929$.

947 Cavada, C., \& Goldman $\square$ Rakic, P. S. (1989). Posterior parietal cortex in rhesus monkey: I. Parcellation 948 of 

Neurology, 287(4), 393-421.

952 Cai, X., \& Padoa-Schioppa, C. (2014). Contributions of orbitofrontal and lateral prefrontal cortices to 953 economic choice and the good-to-action transformation. Neuron, 81(5), 1140-1151.

954 Churchland, M. M., Cunningham, J. P., Kaufman, M. T., Foster, J. D., Nuyujukian, P., Ryu, S. I., Shenoy,

955 K. V., \& Shenoy, K. V. (2012). Neural population dynamics during reaching. Nature, 487(7405),

$956 \quad 51-56$.

957 Cornelissen, F. W., Peters, E. M., \& Palmer, J. (2002). The Eyelink Toolbox: Eye tracking with

958 MATLAB and the Psychophysics Toolbox. Behavior Research Methods, Instruments, and

959 Computers.

960 Dal Monte, O., Chu, C. C. J., Fagan, N. A., \& Chang, S. W. C. (2020). Specialized medial prefrontal-

961 amygdala coordination in other-regarding decision preference. Nature Neuroscience.

962 Dean, H. L., Crowley, J. C., \& Platt, M. L. (2004). Visual and saccade-related activity in macaque

963 posterior cingulate cortex. Journal of neurophysiology, 92(5), 3056-3068.

964 Dean, H. L., \& Platt, M. L. (2006). Allocentric spatial referencing of neuronal activity in macaque

965 posterior cingulate cortex. Journal of Neuroscience.

966 Farashahi, S., Azab, H., Hayden, B., \& Soltani, A. (2018). On the flexibility of basic risk attitudes in 967 monkeys. Journal of Neuroscience, 38(18), 4383-4398.

968 Granger, C. W. J. (1969). Investigating Causal Relations by Econometric Models and Cross-Spectral

969 Methods."Econometrica. Vol. 37, 1969, pp. 424-459.

970

971

972

973

974

975

976

977

Haber, S. N., Kim, K. S., Mailly, P., \& Calzavara, R. (2006). Reward-related cortical inputs define a large striatal region in primates that interface with associative cortical connections, providing a substrate for incentive-based learning. Journal of Neuroscience, 26(32), 8368-8376.

Hare, T. A., Schultz, W., Camerer, C. F., O’Doherty, J. P., \& Rangel, A. (2011). Transformation of stimulus value signals into motor commands during simple choice. Proceedings of the National 
979 Hayden, B., Smith, D. V., \& Platt, M. (2010). Cognitive control signals in posterior cingulate cortex.

$980 \quad$ Frontiers in human neuroscience, 4, 223.

981 Hayden, B. Y., \& Moreno-Bote, R. (2018). A neuronal theory of sequential economic choice. Brain and $982 \quad$ Neuroscience Advances.

983 Hayden, B. Y., Smith, D. V., \& Platt, M. L. (2009). Electrophysiological correlates of default-mode 984 processing in macaque posterior cingulate cortex. Proceedings of the National Academy of $985 \quad$ Sciences, 106(14), 5948-5953.

986 Hayden, B. Y., Nair, A. C., McCoy, A. N., \& Platt, M. L. (2008). Posterior Cingulate Cortex Mediates 987 Outcome-Contingent Allocation of Behavior. Neuron, 60(1), 19-25.

988 Hayden, B. Y., \& Heilbronner, S. R. (2014). All that glitters is not reward signal. Nature neuroscience, $989 \quad 17(9), 1142-1144$.

990 Heilbronner, S. R., \& Platt, M. L. (2013). Causal evidence of performance monitoring by neurons in 991 posterior cingulate cortex during learning. Neuron, 80(6), 1384-1391.

992 Heilbronner, S. R., \& Hayden, B. Y. (2016). Dorsal anterior cingulate cortex: a bottom-up view. Annual 993 review of neuroscience, $39,149-170$.

994 Heilbronner, S. R. (2017). Modeling risky decision-making in nonhuman animals: shared core features.

995 Current opinion in behavioral sciences, 16, 23-29.

996 Heilbronner, S. R., \& Hayden, B. Y. (2016). The description-experience gap in risky choice in nonhuman 997 primates. Psychonomic bulletin \& review, 23(2), 593-600.

998 Kable, J. W., \& Glimcher, P. W. (2007). The neural correlates of subjective value during intertemporal 999 choice. Nature Neuroscience, 10(12), 1625-1633.

1000 Kaplan, R., Schuck, N. W., \& Doeller, C. F. (2017). The Role of Mental Maps in Decision-Making. $1001 \quad$ Trends in Neurosciences, 40(5), 256-259.

1002 Kobayashi, Y., \& Amaral, D. G. (2003). Macaque monkey retrosplenial cortex: II. Cortical afferents. 1003 Journal of Comparative Neurology, 466(1), 48-79. 
1004 Leech, R., \& Sharp, D. J. (2014). The role of the posterior cingulate cortex in cognition and disease.

$1005 \quad$ Brain, 137(1), 12-32.

1006 Levy, D. J., \& Glimcher, P. W. (2012). The root of all value: a neural common currency for

1007 choice. Current opinion in neurobiology, 22(6), 1027-1038.

1008

1009 Luk, C. H., \& Wallis, J. D. (2013). Choice coding in frontal cortex during stimulus-guided or action1010 guided decision-making. Journal of Neuroscience, 33(5), 1864-1871.

1011 Lütkepohl, H. (2007). New Introduction to Multiple Time Series Analysis. New York, NY: Springer-

$1012 \quad$ Verlag.

1013 MacKay, D. J. C. (2003). Information Theory, Inference and Learning Algorithms. Cambridge University

$1014 \quad$ Press.

1015 Mante, V., Sussillo, D., Shenoy, K. V., \& Newsome, W. T. (2013). Context-dependent computation by

1016 recurrent dynamics in prefrontal cortex. Nature, 503(7474), 78-84.

1017 Morecraft, R. J., Cipolloni, P. B., Stilwell-Morecraft, K. S., Gedney, M. T., \& Pandya, D. N. (2004).

1018 Cytoarchitecture and Cortical Connections of the Posterior Cingulate and Adjacent Somatosensory

1019 Fields in the Rhesus Monkey. Journal of Comparative Neurology.

1020 Morecraft, R. J., Geula, C., \& Mesulam, M. M. (1992). Cytoarchitecture and neural afferents of

1021 orbitofrontal cortex in the brain of the monkey. Journal of Comparative Neurology, 323(3), 341-

1022358.

1023 Mufson, E. J., \& Pandya, D. N. (1984). Some observations on the course and composition of the

1024 cingulum bundle in the rhesus monkey. Journal of Comparative Neurology, 225(1), 31-43.

1025 Murray, J. D., Bernacchia, A., Roy, N. A., Constantinidis, C., Romo, R., \& Wang, X. J. (2017). Stable

1026 population coding for working memory coexists with heterogeneous neural dynamics in prefrontal

1027 cortex. Proceedings of the National Academy of Sciences of the United States of America, 114(2),

$1028 \quad 394-399$.

1029 Murray, E. A., \& Rudebeck, P. H. (2018). Specializations for reward-guided decision-making in the 
primate ventral prefrontal cortex. Nature Reviews Neuroscience, 19(7), 404-417.

1031 Niv, Y. (2019). Learning task-state representations. Nature neuroscience, 22(10), 1544-1553.

1032 Noonan, M. P., Walton, M. E., Behrens, T. E. J., Sallet, J., Buckley, M. J., \& Rushworth, M. F. S.

1033 (2010). Separate value comparison and learning mechanisms in macaque medial and lateral

1034 orbitofrontal cortex. Proceedings of the National Academy of Sciences, 107(47), 20547-20552.

1035 O'Doherty, J. P. (2014). The problem with value. Neuroscience \& Biobehavioral Reviews, 43, $259-268$.

1036 Olson, C. R., Musil, S. Y., \& Goldberg, M. E. (1996). Single neurons in posterior cingulate cortex of

1037 behaving macaque: Eye movement signals. Journal of Neurophysiology, 76(5), 3285-3300.

1038 Öngür, D., \& Price, J. L. (2000). The organization of networks within the orbital and medial prefrontal

1039 cortex of rats, monkeys and humans. In Cerebral Cortex.

1040 Padoa-Schioppa, C. (2011). Neurobiology of Economic Choice: A Good-Based Model. Annual Review of

$1041 \quad$ Neuroscience, 34(1), 333-359.

1042 Padoa-Schioppa, C., \& Conen, K. E. (2017). Orbitofrontal Cortex: A Neural Circuit for Economic

1043 Decisions. Neuron, 96(4), 736-754.

1044 Pandya, D. N., Van Hoesen, G. W., \& Mesulam, M. M. (1981). Efferent connections of the cingulate

1045 gyrus in the rhesus monkey. Experimental Brain Research.

1046 Pandya, D. N., \& Seltzer, B. (1982). Intrinsic connections and architectonics of posterior parietal cortex in

1047 the rhesus monkey. Journal of Comparative Neurology, 204(2), 196-210.

1048 Parvizi, J., Van Hoesen, G. W., Buckwalter, J., \& Damasio, A. (2006). Neural connections of the

1049 posteromedial cortex in the macaque. Proceedings of the National Academy of Sciences of the

$1050 \quad$ United States of America.

1051 Paxinos G, Huang XF, Petrides M, T. A. (2009). The Rhesus Monkey Brain in Stereotaxic Coordinates.

1052 Academic Press.

1053 Pearson, J. M., Hayden, B. Y., Raghavachari, S., \& Platt, M. L. (2009). Neurons in Posterior Cingulate

1054 Cortex Signal Exploratory Decisions in a Dynamic Multioption Choice Task. Current Biology.

1055 Pesaran, B. (2010). Neural correlations, decisions, and actions. Current Opinion in Neuobiology, 20(2), 
166-171.

1057 Pirrone, A., Azab, H., Hayden, B. Y., Stafford, T., \& Marshall, J. A. (2018). Evidence for the speed-value

$$
\text { trade-off: Human and monkey decision making is magnitude sensitive. Decision, 5(2), } 129 .
$$

Rangel, A., Camerer, C., \& Montague, P. R. (2008). A framework for studying the neurobiology of valuebased decision making. Nature reviews neuroscience, 9(7), 545-556.

Roesch, M. R., Taylor, A. R., \& Schoenbaum, G. (2006). Encoding of time-discounted rewards in orbitofrontal cortex is independent of value representation. Neuron, 51(4), 509-520.

Rudebeck, P. H., \& Murray, E. A. (2011). Balkanizing the primate orbitofrontal cortex: distinct subregions for comparing and contrasting values. Annals of the New York Academy of Sciences, 1239, 1.

Rudebeck, P. H., Saunders, R. C., Lundgren, D. A., \& Murray, E. A. (2017). Specialized representations of value in the orbital and ventrolateral prefrontal cortex: desirability versus availability of outcomes. Neuron, 95(5), 1208-1220.

Schuck, N. W., Cai, M. B., Wilson, R. C., \& Niv, Y. (2016). Human Orbitofrontal Cortex Represents a

Rushworth, M. F. S., Noonan, M. P., Boorman, E. D., Walton, M. E., \& Behrens, T. E. (2011). Frontal cortex and reward-guided learning and decision-making. Neuron, 70(6), 1054-1069.

Scherberger, H., Jarvis, M. R., \& Andersen, R. A. (2005). Cortical Local Field Potential Encodes Cognitive Map of State Space. Neuron, 91(6), 1402-1412.

Sleezer, B. J., Castagno, M. D., \& Hayden, B. Y. (2016). Rule encoding in orbitofrontal cortex and striatum guides selection. Journal of Neuroscience, 36(44), 11223-11237. memory, prospection, navigation, theory of mind, and the default mode: A quantitative meta- 
analysis. Journal of Cognitive Neuroscience, 21(3), 489-510.

1083 Stalnaker, T. A., Cooch, N. K., \& Schoenbaum, G. (2015). What the orbitofrontal cortex does not do.

$1084 \quad$ Nature Neuroscience, 18(5), 620.

1085 Strait, C. E., Blanchard, T. C., \& Hayden, B. Y. (2014). Reward value comparison via mutual inhibition 1086 in ventromedial prefrontal cortex. Neuron, 82(6), 1357-1366.

1087 Strait, C. E., Sleezer, B. J., \& Hayden, B. Y. (2015). Signatures of value comparison in ventral striatum 1088 neurons. PLoS Biol, 13(6), e1002173.

1089 Strait, C. E., Sleezer, B. J., Blanchard, T. C., Azab, H., Castagno, M. D., \& Hayden, B. Y. (2016).

1090 Neuronal selectivity for spatial positions of offers and choices in five reward regions. Journal of 1091 neurophysiology, 115(3), 1098-1111.

1092 Timme, N. M., \& Lapish, C. (2018). A tutorial for information theory in neuroscience. ENeuro, 5(3).

1093 Vickery, T. J., Chun, M. M., \& Lee, D. (2011). Ubiquity and specificity of reinforcement signals

1094 throughout the human brain. Neuron, 72(1), 166-177

1095 Vogt, B. A., \& Paxinos, G. (2014). Cytoarchitecture of mouse and rat cingulate cortex with human 1096 homologies. Brain Structure and Function.

1097 Wallis, J. D. (2007). Orbitofrontal cortex and its contribution to decision-making. Annual Review of $1098 \quad$ Neuroscience, 30(1), 31-56.

1099 Wang, F., Schoenbaum, G., \& Kahnt, T. (2020). Interactions between human orbitofrontal cortex and 1100 hippocampus support model-based inference. PLoS Biology, 18(1), e3000578.

1101 Wang, M. Z., \& Hayden, B. Y. (2017). Reactivation of associative structure specific outcome responses 1102 during prospective evaluation in reward-based choices. Nature Communications, 8(May), 1-13.

1103 Widge, A. S., Heilbronner, S. R., \& Hayden, B. Y. (2019). Prefrontal cortex and cognitive control: new 1104 insights from human electrophysiology. F1000Research, 8.

1105 Wikenheiser, A. M., \& Schoenbaum, G. (2016). Over the river, through the woods: Cognitive maps in the 1106 hippocampus and orbitofrontal cortex. Nature Reviews Neuroscience, 17(8), 513-523.

1107 Wilson, R. C., Takahashi, Y. K., Schoenbaum, G., \& Niv, Y. (2014). Orbitofrontal cortex as a cognitive 
1108 map of task space. Neuron, 81(2), 267-279.

1109 Yim, M. Y., Cai, X., \& Wang, X. J. (2019). Transforming the Choice Outcome to an Action Plan in

1110 Monkey Lateral Prefrontal Cortex: A Neural Circuit Model. Neuron, 103(3), 520-532.e5.

1111 Yoo, S. B. M., Sleezer, B. J., \& Hayden, B. Y. (2018). Robust encoding of spatial information in

1112 orbitofrontal cortex and striatum. Journal of Cognitive Neuroscience, 30(6), 898-913.

1113 Yoo, S. B. M., \& Hayden, B. Y. (2020). The transition from evaluation to selection involves neural

1114 subspace reorganization in core reward regions. Neuron, 105(4), 712-724.

1115 Yoo, S. B. M., \& Hayden, B. Y. (2018). Economic choice as an untangling of options into actions.

$1116 \quad$ Neuron, 99(3), 434-447.

1117 Young, M. E., \& Mccoy, A. W. (2015). A delay discounting task produces a greater likelihood of waiting

1118 than a deferred gratification task. Journal of the Experimental Analysis of Behavior, 103(1), 180-

1119195. 